\title{
SOLVING OF SPECTRAL PROBLEMS FOR CURL AND STOKES OPERATORS
}

\section{R.S. SAKS}

Abstract. In the work we explicitly solve the spectral problems for curl, gradientdivergence, and Stokes operators in a ball $B$ of radius $R$. The eigenfunctions $\mathbf{u}_{\kappa}^{ \pm}$of the curl associated with non-zero eigenvalues $\pm \lambda_{\kappa}$ are expressed by explicit formulas, as well as the vector-functions $\mathbf{q}_{\kappa}$ associated with the zero eigenvalue,

$$
\operatorname{rot} \mathbf{u}_{\kappa}^{ \pm}= \pm \lambda_{\kappa} \mathbf{u}_{\kappa}^{ \pm}, \quad \psi_{n}\left( \pm \lambda_{\kappa} R\right)=0,\left.\quad \mathbf{n} \cdot \mathbf{u}_{\kappa}^{ \pm}\right|_{S}=0 ; \quad \operatorname{rot} \mathbf{q}_{\kappa}=0,\left.\quad \mathbf{n} \cdot \mathbf{q}_{\kappa}\right|_{S}=0,
$$

where

$$
\psi_{n}(z)=(-z)^{n}\left(\frac{d}{z d z}\right)^{n} \frac{\sin z}{z}, \quad \kappa=(n, m, k), n \geq 0, m \in \mathbb{N},|k| \leqslant n
$$

The same vector-functions are the eigenfunctions for the gradient-divergence operator with other eigenvalues,

$$
\nabla \operatorname{div} \mathbf{u}_{\kappa}^{ \pm}=0 ; \quad \nabla \operatorname{div} \mathbf{q}_{\kappa}=\mu_{\kappa} \mathbf{q}_{\kappa}, \quad \mu_{\kappa}=\left(\alpha_{n, m} / R\right)^{2}, \quad \psi_{n}^{\prime}\left(\alpha_{n, m}\right)=0 .
$$

The constructed system of vector eigenfunctions is complete and orthogonal in space $\mathbf{L}_{2}(B)$.

The eigenfunctions $\left(\mathbf{v}_{\kappa}, p_{\kappa}\right)$ of the Stokes operator in the ball are represented as a sum of two eigenfunctions of the curl associated with opposite eigenvalues: $\mathbf{v}_{\kappa}=\mathbf{u}_{\kappa}^{+}+\mathbf{u}_{\kappa}^{-}$, $p_{\kappa}=$ const.

Keywords: curl, gradient-divergence, and Stokes operators, eigenvalues, eigenfunctions, Fourier series.

Mathematics Subject Classification: 35P05, 35P10.

\section{INTRODUCTION}

1.1. Formulation of problem. Let $G$ be a bounded domain in $R^{3}$ with a piecewise smooth boundary $\Gamma, \mathbf{n}$ be the outward normal to $\Gamma$. In particular, $G$ can be a ball $B,|x|<R$, with boundary $S$.

Problem 1. Find all the eigenvalues $\lambda$ and the eigenfunctions $\mathbf{u}(\mathbf{x})$ in $\mathbf{L}_{2}(G)$ for the curl operator such that

$$
\begin{gathered}
\operatorname{rot} \mathbf{u}=\lambda \mathbf{u} \quad \text { in } \quad G, \\
\left.\mathbf{n} \cdot \mathbf{u}\right|_{\Gamma}=0,
\end{gathered}
$$

where $\mathbf{n} \cdot \mathbf{u}$ is the scalar product of the vectors $\mathbf{u}$ and $\mathbf{n}$.

As the domain $\mathcal{M}_{\mathcal{R}}$ of the operator $\mathcal{R}$ in Problem 1, we choose all the vector-functions $\mathbf{v}(\mathbf{x})$ in the class $\mathcal{C}^{2}(G) \cap \mathcal{C}(\bar{G})$ satisfying boundary condition (2) and condition $\operatorname{rot} \mathbf{v} \in \mathbf{L}_{2}(G)$.

The space of test vector-functions $\mathbf{D}(G)$ is included into $\mathcal{M}_{\mathcal{R}}$ and is dense in $\mathbf{L}_{2}(G)$ [3].

Thus, the problem is to find the values $\lambda$ for which equation (1) has nonzero solutions $\mathbf{u}(\mathbf{x})$ in the domain $\mathcal{M}_{\mathcal{R}}$, i.e., to determine the pair $(\lambda, \mathbf{u})$ of an eigenvalue $\lambda$ and an eigenfunctions $\mathbf{u} \neq 0$.

R.S. Saks, Solving of SPeCtral Problems for CuRl and Stokes operators.

(c) SAKS R.S. 2013.

Submitted January 12, 2012. 
1.2. On applications. The eigenfunctions of Problem 1 have application in hydrodynamics, where they are called the Beltrami fields [9], in celestial mechanics and in the physics of plasma they are called the force-free fields (see S. Chandrasekhar [11] and J.B. Taylor [12]).

By Taylor's theory, on the force-free fields, in the last stable equilibrium before decay, the speed of the plasma in tokamaks is $\mathbf{u}(\mathbf{x})$, for which $\operatorname{rot} \mathbf{u}=\lambda \mathbf{u}$ and $\lambda=$ const.

According to S. Chandrasekhar, outside the photosphere of a star, the magnetic field $\mathbf{H}$ is so that the Lorentz force $L$ proportional to the cross-product $[\operatorname{rot} \mathbf{H}, \mathbf{H}]$ disappears.

By Arnold theorem [13] 1965, almost all current-flow lines of the ideal liquid reel on either cylinders or tori. At that, stationary flows with the speed $\mathbf{v}(\mathbf{x})$ satisfying the condition $[\operatorname{rot} \mathbf{v}, \mathbf{v}]=0$ are excluded from the consideration. The flows with the speed $\mathbf{v}(\mathbf{x})$ obeying equation (1) obviously satisfy this condition. Referring to the calculations of M. Henon [14], V. Arnold writes that "such flows can have current-flow lines with rather complicated topology typical for the problems in celestial mechanics."

In 1970, the author studied the boundary value problems for non-elliptic system

$$
\operatorname{rot} \mathbf{u}+\lambda \mathbf{u}=\mathbf{f}
$$

in a bounded domain $G$ with a smooth boundary and proved that for each $\lambda \neq 0$, the system has Fredholm solvable boundary value problems with a non-zero index [17], [18]. The problem of this kind is that with the boundary condition

$$
\left.\mathbf{n} \cdot \mathbf{u}\right|_{\Gamma}=g .
$$

For a ball $B$, a way of explicit solving problem (3), (4) was found (see [19]) and the formulas for the eigenfunctions of the curl as $\lambda \neq 0$ were written as solutions to a homogeneous problem.

The feature of this problem is that the low-order term $\lambda \mathbf{u}$ in system (3) improves essentially its solvability ( see $\S 7$ ).

I published this result (formulae (36), (37)) in 2000 [21], when I learnt about the applications and work by S. Chandrasekhar and P.S. Kendall [22] 1957 suggested another approach for solving spectral problem 1 in a ball and cylinder.

In a ball their method does not work, and in a cylinder it was performed by D. Montgomery, L. Turner, G. Vahala [23] 1978, who suggested to employ the eigenfunctions of the curl in studying a turbulence in a plasma.

Self-adjoint extensions of the operator in Problem 1 were studied by P.E. Berhin [24] 1975, Y. Giga, Z. Yoshida [25] 1990, and R. Picard [26] 1996.

For other aspects of the theory see book by V.V. Kozlov [4] and the reviews by V.V. Pukhnachev [9] and by A. Makhalov and V. Nikolaenko [28].

In 2003, O.A. Ladyzhesnkaya solved the problem "On constructing the basis in the space of solenoidal fields" [1] and interested in a possibility of explicit calculating the eigenfunctions for the Stokes operator in simplest domains.

It happened [16] that in the periodic case the vector eigenfunctions $\left(\mathbf{v}_{k}, p_{k}\right)$ of the Stokes operator are so that $\nabla p_{k}=0$ and the vector-functions $\mathbf{v}_{k}$ coincide with the solenoidal eigenfunctions of the curl $\mathbf{u}_{k}^{ \pm}$as $k \neq 0$ and $\mathbf{u}_{0}^{j}$ as $k=0$.

On their basis, global solutions to the Navier-Stokes equations in a uniformly rotating space were constructed [29] and the equations describing the interaction between basis vortical flows were found [30].

Later [15], the author succeeded to calculated the eigenfunctions $\left(\mathbf{v}_{n}, p_{n}\right)$ of the Stokes operator in a ball subject to the condition $\left.\mathbf{v}_{n}\right|_{S}=0$. In this case, each vector eigenfunction $\mathbf{v}_{n}$ of the Stokes operator is the sum, $\mathbf{v}_{n}=\mathbf{u}_{n}^{+}+\mathbf{u}_{n}^{-}$, of the vector eigenfunctions $\mathbf{u}_{n}^{ \pm}$of the curl associated with opposite eigenvalues and $p_{n}=$ const. (see $\S 6$ ).

1.3. Structure of work and main results. In Section 1, the solving of Problem 1 in a ball as $\lambda \neq 0$ is reduced to the solving of the spectral Dirichlet problem for the scalar Laplace 
operator subject to the condition $v(0)=0$ at the center of the ball that is solved explicitly in Section 2. Its eigenvalues are determined by the zeroes of the Bessel functions of a halfinteger order, and the eigenfunctions are the products of the Bessel functions and the spherical functions.

In Section 3, we give explicit formulae for nonzero eigenvalues $\pm \lambda_{n, m}$ and the eigenfunctions $\mathbf{q}_{n, m, k}^{ \pm}(\mathbf{x})$ of the curl operator in a ball. Formulas (36), 37) were published in [21], while formulas (43) are published here for the first time. They give a possibility to calculate the speed distribution of the liquid flow $\mathbf{q}_{n, m, k}^{ \pm}(\mathbf{x})$ inside a ball and conceive of the motion of such flow.

In Section 4, the spectral problem for the gradient-divergence operator is reduced to the solution of the Neumann problem for the scalar Laplace operator whose solutions are known. We provide formulae (53) for the eigenfunctions $\mathbf{q}_{n, m, k}(\mathbf{x})$ of the curl operator in a ball associated with the zero eigenvalue. These formulae are published here for the first time.

In Section 5, we prove that the constructed family of the eigenfunctions

$$
\left\{\mathbf{q}_{n, m, k}(\mathbf{x}), \mathbf{q}_{n, m, k}^{+}(\mathbf{x}), \mathbf{q}_{n, m, k}^{-}(\mathbf{x})\right\} \quad n \geq 0, m \in \mathbb{N},|k| \leqslant n,
$$

for the curl operator is orthogonal and complete in the space $\mathbf{L}_{2}(B)$ that consists of the square integrable vector functions $\mathbf{f}$. This family makes an orthonormal basis in $\mathbf{L}_{2}(B)$.

We provide an analogue of Weil expansion [10] of a vector field $\mathbf{f}$ in $\mathbf{L}_{2}(B)$ (with zero component $\left.\mathbf{n} \cdot \mathbf{f}\right|_{S}=0$ ) into a curlfree field $\mathbf{a}$ and a solenoidal field $\mathbf{b}, \mathbf{f}(\mathbf{x})=\mathbf{a}(\mathbf{x})+\mathbf{b}(\mathbf{x})$.

In Section 6, we determine the relation between the solutions of the spectral problems for the curl and Stokes operator and give explicitly the solutions to the spectral problem for the Stokes operator in a ball. Formulae (93) for the eigenfunctions of the Stokes operator are published here for the first time.

In Section 7, as an example, we provide the solving of the boundary value problem (2), (3) by the Fourier method in two cases: as $\lambda \neq 0, \pm \lambda_{n, m}$ and as $\lambda=0$. We note that as $\lambda=0$, the solvability of the problem worsens and its kernel becomes infinite-dimensional.

1.4. Study of operator in problem. As $\lambda \neq 0$, systems $(3)$ and

$$
\nabla \operatorname{div} \mathbf{u}+\lambda \mathbf{u}=\mathbf{f}
$$

are elliptic by Vainberg and Grushin [6]. The first order operator rot $+\lambda \mathrm{I}$ is not elliptic since the rank of its symbolic matrix $\sigma_{1}$ (rot $)(\xi)$ equals two for each $\xi \in \mathcal{R}^{3} \backslash 0$ and is less than three [20.

Relation div rot $\mathbf{u} \equiv 0$ for each smooth vector function $\mathbf{u}$ and system of equations (1) as $\lambda \neq 0$ imply that $\operatorname{div} \mathbf{u}=0$. Hence, $\mathbf{u}(\mathbf{x})$ solves the elliptic system

$$
\operatorname{rot} \mathbf{u}-\lambda \mathbf{u}=0, \quad \operatorname{div} \mathbf{u}=0 .
$$

Such operator, rot $+\lambda I$, is called the reducible to an elliptic operator [6].

It is easy to check that system (6) and boundary condition (2) is an overdetermined elliptic boundary value problem is the sense of the theory by V.A. Solonnikov [7]. It follows from the relation

$$
(\operatorname{rot}+\lambda I)(\operatorname{rot}-\lambda I) \mathbf{u}=-\Delta \mathbf{u}+\nabla \operatorname{div} \mathbf{u}-\lambda^{2} \mathbf{u}
$$

that as $\lambda \neq 0$, a solution $\mathbf{u} \in \mathcal{C}^{2}(B)$ to equation (1) solves also the second order elliptic system

$$
-\Delta \mathbf{u}=\lambda^{2} \mathbf{u}, \quad \operatorname{div} \mathbf{u}=0 .
$$

Moreover, there is a one-to-one correspondence between the solutions $\mathbf{u}$ of problem (3), (4) and the solutions $(\mathbf{u}, q)$ to the elliptic boundary value problem

$$
\operatorname{rot} \mathbf{u}+\lambda \mathbf{u}+\nabla q=\mathbf{f}, \quad \lambda \operatorname{div} \mathbf{u}=\operatorname{div} \mathbf{f},\left.\quad \mathbf{n} \cdot \mathbf{u}\right|_{\Gamma}=g,\left.\quad q\right|_{\Gamma}=0
$$

where $q=0$ in $G$. 
In accordance with the theory of elliptic boundary value problems, for problem (9) in a bounded domain $G$ with a smooth boundary $\Gamma$, the estimate

$$
C_{s}\|\mathbf{u}\|_{s+1} \leqslant\|\operatorname{rot} \mathbf{u}\|_{s}+\|\operatorname{div} \mathbf{u}\|_{s}+|\mathbf{n} \cdot \mathbf{u}|_{s+1 / 2}+\|\mathbf{u}\|_{s}
$$

holds true for a vector function $\mathbf{u}$ in the Sobolev space $\mathbf{H}^{s+1}(G) \equiv \mathbf{W}_{2}^{s+1}(G)$, where $C_{s}$ is a positive constant, $\mathbf{n} \cdot \mathbf{u}$ is the trace of the normal component of $\mathbf{u}$ on $\Gamma$, and $|\mathbf{n} \cdot \mathbf{u}|_{s+1 / 2}$ is its norm in $H^{s+1 / 2}(\Gamma), s \geq 0$ (see [7], [8], [20], 25]).

This theory implies that as $\lambda \neq 0$

a) the number of linear independent solutions to Problem 1 is finite,

b) each (generalized) solution to the problem is infinitely differentiable up to the boundary if the boundary is infinitely differentiable.

1.5. Reduction of Problem 1 to Dirichlet spectral problem in ball. While constructing the eigenfunctions associated with nonzero eigenvalues of the curl operator in a ball $B$, we arrive at the following Dirichlet problem for the Laplace operator.

Problem 2. Find the eigenvalues $\mu$ and the eigenfunctions $v(x)$ of the scalar Laplace operator $-\Delta$ such that

$$
-\Delta v=\mu v \quad \text { in } \quad B,\left.\quad v\right|_{S}=0, \quad v(0)=0 .
$$

As the domain $\mathcal{M}_{\mathcal{L}_{1}}$ of the operator $\mathcal{L}_{1}$ in Problem 2, we choose the functions $v(\mathbf{x})$ in $\mathcal{C}^{2}(B) \cap \mathcal{C}(\bar{B})$ satisfying the conditions $\left.v\right|_{S}=0, v(0)=0$, and $\Delta v \in L_{2}(B)$.

We denote by $v(\mathbf{x})=\mathbf{x} \cdot \mathbf{u}=r u_{r}$ the scalar product of the vectors $\mathbf{x}$ and $\mathbf{u}$. The following lemma holds true.

Lemma 1. As $\lambda \neq 0$, for each solution $(\lambda, \mathbf{u})$ of Problem 1 in the ball $B,\left(\lambda^{2}, \mathbf{x} \cdot \mathbf{u}\right)$ is a solution to Problem 2.

Indeed, by (8), (2), and the boundedness of $\mathbf{u}$ in a vicinity of zero we have

$$
-\Delta v=-\mathbf{x} \cdot \Delta \mathbf{u}-2 \operatorname{div} \mathbf{u}=\lambda^{2} v,\left.\quad v\right|_{S}=\left.R u_{r}\right|_{r=R}=0, \quad v(0)=\left.r u_{r}\right|_{r=0}=0 .
$$

\section{Solving OF SPECTRAL PROBlem 2.}

2.1. Zeroes of functions $\psi_{n}(z)$. Let $\rho_{m, n}>0$ be the Bessel functions of half-integer order, i.e., $J_{n+\frac{1}{2}}\left(\rho_{m, n}\right)=0$, where $n \geq 0, m=1,2, \ldots$ They are also the zeroes of the functions

$$
\psi_{n}(z) \equiv \sqrt{\frac{\pi}{2 z}} J_{n+\frac{1}{2}}(z)=\sqrt{\frac{\pi}{2 z}} \sum_{p=0}^{\infty} \frac{(-1)^{p}}{p ! \Gamma\left(n+1+p+\frac{1}{2}\right)}\left(\frac{z}{2}\right)^{n+2 p+\frac{1}{2}},
$$

As L. Euler showed (see [3], Section 23, P. 356), the cylindrical functions $J_{n+\frac{1}{2}}(z)$ of half-integer order are expressed in terms of the elementary functions, namely,

$$
\psi_{n}(z)=(-z)^{n}\left(\frac{d}{z d z}\right)^{n}\left(\frac{\sin z}{z}\right) .
$$

It implies that

$$
\psi_{n}(-z)=(-1)^{n} \psi_{n}(z)
$$

and that the zeroes of the functions $\psi_{n}(z)$ are located on the real axis symmetrically w.r.t. the point $z=0$. 
2.2. Dirichlet spectral problem. It is solved by the separations of variables in spherical coordinates $(r, \theta, \varphi)$. By $L$ we denote the operator in the problem. In the textbook by V.S. Vladimirov [3, Section 26], it was shown that the eigenvalues of the Laplace operator $\mathcal{L}$ in the ball $B$ are $\lambda_{n, m}^{2}$, where $\lambda_{n, m}=\rho_{n, m} R^{-1}, n \geq 0, m \in N$, and the numbers $\rho_{n, m}>0$ are the zeroes of the functions $\psi_{n}(z)$,

the real eigenfunctions $v_{\kappa}$ associated with eigenvalues $\lambda_{n, m}^{2}$ read as follows,

$$
v_{\kappa}(r, \theta, \varphi)=c_{\kappa} \psi_{n}\left(\lambda_{n, m} r\right) Y_{n}^{k}(\theta, \varphi),
$$

where $\kappa=(n, m, k)$ is a multi-index, $n \geq 0,|k| \leq n, m \in \mathbb{N}, c_{\kappa}$ are arbitrary real constants, $P_{n}^{k}(\cos \theta)$ are the associated Legendre functions, $0<r \leq R, 0 \leq \theta \leq \pi, Y_{n}^{k}(\theta, \varphi)$ are real spherical functions $0 \leq \varphi \leq 2 \pi$. They are

$$
Y_{n}^{k}(\theta, \varphi)=\left\{\begin{aligned}
P_{n}^{k}(\cos \theta) \cos (k \varphi), & \text { if } \quad k=0,1, \ldots, n \\
P_{n}^{|k|}(\cos \theta) \sin (|k| \varphi), & \text { if } \quad k=-1, \ldots,-n
\end{aligned}\right.
$$

As $n=0,1,2$, the functions $Y_{n}(\theta, \varphi)=\sum_{k=-n}^{n} a_{k n} Y_{n}^{k}(\theta, \varphi)$ read as follows,

$$
\begin{gathered}
Y_{0}=a_{00}, Y_{1}=a_{01} \cos \theta+\left(a_{11} \cos \varphi+a_{-1,1} \sin \varphi\right) \sin \theta, \\
Y_{2}=a_{02}\left(3 \cos ^{2} \theta-1\right)+\left(a_{12} \cos \varphi+a_{-1,2} \sin \varphi\right) \sin \theta \cos \theta+\left(a_{22} \cos 2 \varphi+a_{-2,2} \sin 2 \varphi\right) \sin ^{2} \theta .
\end{gathered}
$$

By the definition of the spherical functions, the product $r^{n} Y_{n}^{k}(\theta, \varphi)$ is a homogeneous harmonic polynomial of order $n$ w.r.t. $x_{1}, x_{2}, x_{3}$. It follows from formulae (15), (13) that the functions $v_{\kappa}(x)$ belong to the class $C^{\infty}(B)$ in a ball $B$ of an arbitrary radius $R>0$.

The orthogonality and completeness of the Bessel functions in $L_{2}[(0, R) ; r]$ and of the spherical functions in $L_{2}\left(S_{1}\right)$ yield that for different $\kappa=(n, m, k)$, the functions $v_{\kappa}$ are orthogonal in $L_{2}(B)$.

The system of the functions $\left\{v_{\kappa}\right\}$ is complete in $L_{2}(B)[3]$. Being normalized by the condition

$$
\begin{gathered}
\int_{B} v_{\kappa^{\prime}} v_{\kappa} d \mathbf{x}= \\
=a_{\kappa^{\prime}} a_{\kappa} \int_{0}^{R} \psi_{n^{\prime}}\left(\rho_{n^{\prime}, m^{\prime}} r / R\right) \psi_{n}\left(\rho_{n, m} r / R\right) r^{2} d r \int_{0}^{\pi} \int_{0}^{2 \pi} Y_{n^{\prime}}^{k^{\prime}}(\theta, \varphi) Y_{n}^{k}(\theta, \varphi) \sin \theta d \theta d \varphi=\delta_{\kappa^{\prime}, \kappa},
\end{gathered}
$$

it makes an orthonormal basis in $L_{2}(B)$. The normalizing factors $a_{\kappa}$ are such that

$$
\left(a_{n, m, k}\right)^{-1}=R\left|J_{n+1 / 2}^{\prime}\left(\rho_{n, m}\right)\right| \sqrt{\pi \frac{1+\delta_{0 k}}{2 n+1} \frac{(n+|k|) !}{(n-|k|) !}} .
$$

2.3. Equivalent integral equation. It was shown in the book [3, Section 29] that if $f \in$ $C^{1}(B) \cap C(\bar{B})$, the boundary value problem

$$
-\Delta v=\mu v+f(x),\left.\quad v\right|_{S}=0, \quad v \in C^{2}(B) \cap C(\bar{B}),
$$

is equivalent to the integral equation

$$
v(x)=\int_{B} G(x, y)[\mu v(y)+f(y)] d y, \quad v \in C(\bar{B}),
$$

with the symmetric weakly polar kernel

$$
G(x, y)=\frac{1}{4 \pi|x-y|}-\frac{R|y|}{\left.4 \pi|x| y\right|^{2}-y R^{2} \mid} .
$$

As the domain $\mathcal{M}_{\mathcal{L}}$ of the operator $\mathcal{L}$ in Problem $[20$, one chooses [3] the functions $v$ in the class $\mathcal{C}^{2}(B) \cap \mathcal{C}(\bar{B})$ satisfying the boundary condition $\left.v\right|_{S}=0$ and the belonging $\Delta v \in L_{2}(B)$.

The eigenvalues and the eigenfunctions of the operator $\mathcal{L}$ coincide with the characteristic numbers and the associated eigenfunctions of the kernel $G(x, y)$. 
According to the theory of integral equations, the set of all the eigenvalues of the Laplace operator $\mathcal{L}$ has no finite accumulating points and each eigenvalue is of finite multiplicity. Each function in $\mathcal{M}_{\mathcal{L}}$ can be expanded into a regular convergent Fourier series in terms of the eigenfunctions of the operator $\mathcal{L}$.

Therefore, all the eigenvalues $\lambda_{n, m}^{2}=\rho_{n, m}^{2} R^{-2}$ of the operator $\mathcal{L}$ can be taken in the ascending order

$$
0<\mu_{1} \leqslant \mu_{2} \leqslant \ldots, \quad \mu_{l} \rightarrow \infty, \quad l \rightarrow \infty
$$

counting $\mu_{l}$ in accordance with its multiplicity (the number $\lambda_{n, m}^{2}$ is counted $2 n+1$ times). The associated eigenfunctions are denoted by $V_{1}, V_{2}, \ldots$, so that each eigenvalue $\mu_{l}$ in series (23) is associated with the eigenfunction $V_{l}(x)$,

$$
\mathcal{L} V_{l}=\mu_{l} V_{l}, \quad l=1,2, \ldots, \quad V_{l} \in \mathcal{M}_{\mathcal{L}} .
$$

We choose the eigenfunctions $V_{l}(x)$ being real and orthonormalized,

$$
\left(\mathcal{L} V_{l}, V_{m}\right)=\mu_{l}\left(V_{l}, V_{m}\right)=\mu_{l} \delta_{l m} .
$$

Each function $f(x)$ in $\mathcal{M}_{\mathcal{L}}$ is expanded into the Fourier series in terms of the orthonormal system $\left\{V_{l}(x)\right\}$,

$$
f(x)=\sum_{l=1}^{\infty}\left(f, V_{l}\right) V_{l}(x) .
$$

This series converges in $L_{2}(B)$, and by Hilbert-Schmidt theorem the series regularly converges on $\bar{B}$ (see [3] $\S 20.1$ ). But the set $\mathcal{M}_{\mathcal{L}}$ is dense in $L_{2}(B)$. It implies the completeness of the system $\left\{V_{l}(x)\right\}$ in $L_{2}(B)$. We note that $\left\{V_{l}(x)\right\}$ is the systen $\left\{v_{\kappa}(x)\right\}$ with the aforementioned ordering.

We shall write series (26) (and other similar series) as

$$
f(\mathbf{x})=\sum_{n=0}^{\infty} \sum_{m=1}^{\infty} \sum_{k=-n}^{n}\left(f, v_{n, m, k}\right) v_{n, m, k}(\mathbf{x}) \equiv \sum_{\kappa}\left(f, v_{\kappa}\right) v_{\kappa}(\mathbf{x}),
$$

assuming that the summation of series (27) is made w.r.t. $n, m$ obeying $0<\rho_{n, m}<N$ where $N \rightarrow \infty$.

2.4. Convergence of series in Sobolev space $H^{s}(B)$. According to Theorems 8 and 9 in [5. Chapter 4], for a ball we have the following.

A function $f$ is expanded into Fourier series (27) in terms of the eigenfunctions of the Dirichlet problem in a ball and this series converges in the Sobolev space $H^{s}(B)$ if and only if $f$ belongs to

$$
H_{\mathcal{D}}^{s}(B)=\left\{f \in H^{s}(B):\left.f\right|_{S}=0, \ldots,\left.\triangle^{\sigma} f\right|_{S}=0\right\}, \quad \text { where } \quad \sigma=[(s-1) / 2], \quad s \geq 1 .
$$

If $f \in H_{\mathcal{D}}^{s}(B)$, the series

$$
\sum_{\kappa}\left(f, v_{\kappa}\right)^{2} \lambda_{\kappa}^{2 s}
$$

converges and there exists a positive constant $C>0$ independent of $f$ such that

$$
\sum_{\kappa}\left(f, v_{\kappa}\right)^{2} \lambda_{\kappa}^{2 s} \leqslant C\|f\|_{H^{s}(B)}^{2} .
$$

If $s \geq 2$, each function $f$ in $H_{\mathcal{D}}^{s}(B)$ is expanded into Fourier series (27) converging in the space $\mathcal{C}^{s-2}(B)$. 
2.5. Solving of Problem 2. Since $\psi_{0}(0)=1$, as $\kappa=(0, m, 0)$, the functions $\left\{v_{\kappa}\right\}$ satisfies the condition $v_{\kappa}(0)=0$ of Problem 2 if and only if the corresponding coefficients $c_{(0, m, 0)}$ vanish. It implies

Theorem 1. The eigenvalues $\mu_{n, m}$ of Problem 2 equal $\lambda_{n, m}^{2}$, where $\lambda_{n, m}=\rho_{n, m} R^{-1}$, and the numbers $\rho_{n, m}$ are zeroes of the functions $\psi_{n}(z), m, n \in \mathbb{N}$. The eigenfunctions $v_{\kappa}$ associated with $\lambda_{n, m}^{2}$ read as follows,

$$
v_{\kappa}(r, \theta, \varphi)=c_{\kappa} \psi_{n}\left(\lambda_{n, m} r\right) Y_{n}^{k}(\theta, \varphi),
$$

where $m, n \in \mathbb{N}$ and $|k| \leq n, \kappa=(n, m, k)$. The multiplicity of the eigenvalues $\mu_{n, m}$ equals $2 n+1$.

Thus, the spectrum of Problem 2 is discrete and has no finite accumulation points, and the eigenfunctions $v_{\kappa}$ of the problem are expressed in terms of cylindrical and spherical functions.

\section{Solving OF SPECTRAL PROBlem 1 IN BALL}

3.1. Construction of solutions to Problem 1. In addition, we prove that its eigenvalues $\pm \lambda_{n, m}$ are the square roots of the eigenvalues of Problem 2.

Lemma 2. In the ball $B$, as $\mu>0$, to each solution $(\mu, v)$ of Problem 2, there correspond exactly two solutions $\left(\sqrt{\mu}, \mathbf{u}^{+}\right)$and $\left(-\sqrt{\mu}, \mathbf{u}^{-}\right)$of Problem 1 such that $\mathbf{x} \cdot \mathbf{u}^{+}=\mathbf{x} \cdot \mathbf{u}^{-}=v$.

Proof. The proof is based on the representation of the system $\operatorname{rot} \mathbf{u}=\lambda \mathbf{u}$, $\operatorname{div} \mathbf{u}=0$ of four real equations written in the spherical coordinates as the system of two complex equations

$$
\left(\partial_{r}-i \lambda\right) r w=r^{-1} \mathrm{H} v, \quad \mathrm{~K} w=\lambda v-i r^{-1} \partial_{r}(r v)
$$

w.r.t. to the complex-valued function $w=u_{\varphi}+i u_{\theta}$ and the real function $v=r u_{r}$. The operators $\mathrm{H}$ and $\mathrm{K}$ read as follows,

$$
\mathrm{H} v=\left(\sin ^{-1} \theta \partial_{\varphi}+i \partial_{\theta}\right) v, \quad \mathrm{~K} w=\sin ^{-1} \theta\left(\partial_{\theta} \sin \theta+i \partial_{\varphi}\right) w
$$

It is easy to make sure that $-\Delta v=\lambda^{2} v$ is the compatibility condition of equations (32).

Let $(\mu, v)$ be a fixed solution to Problem 2. We find nonzero solutions to the problem as follows. The function $u_{r}$ is defined as the fraction $v / r$. We let $\underline{\lambda}=\sqrt{\mu}$ or $\underline{\lambda}=-\sqrt{\mu}$ and substitute $\underline{\lambda}$ and $\underline{v}=v$ into equation (32). Now their right hand sides are defined and the equations are compatible. We define the functions $u_{\theta}$ and $u_{\varphi}$ by solving these systems. The general solution to the first equation 32 is

$$
\underline{w}=d r^{-1} e^{i \underline{\lambda} r}+r^{-1} \int_{0}^{r} e^{i \underline{\lambda}(r-t)} \mathrm{H} \underline{v}(t, \theta, \varphi) t^{-1} d t
$$

where $d$ is a function of the variables $\varphi$ and $\theta$ being zero if we seek the solution in the Sobolev space $W_{2}^{1}(B)$ or in the class of bounded functions. It remains to check that the function $\underline{w}$ satisfies the second equation in 32 . We obtain

$$
\mathrm{K} \underline{w}=r^{-1} \int_{0}^{r} e^{i \underline{\lambda}(r-t)} \mathrm{KH} \underline{v}(t, \theta, \varphi) t^{-1} d t=r^{-1} \int_{0}^{r} e^{i \underline{\lambda}(r-t)}\left[\sin ^{-1} \theta\left(\partial_{\theta} \partial_{\varphi}-\partial_{\varphi} \partial_{\theta}\right) \underline{v}+i \Delta_{\theta}, \varphi \underline{v}\right] t^{-1} d t,
$$

where $\Delta_{\theta, \varphi}$ is the Laplace-Beltrami operator. We write the Helmholtz equation in the spherical coordinates,

$$
\frac{1}{r \sin \theta}\left[\partial_{\theta}\left(\sin \theta \partial_{\theta}\right)+\frac{1}{\sin \theta}\left(\partial_{\varphi}\right)^{2}\right] v=-\lambda^{2} r v-\frac{1}{r} \partial_{r}\left(r^{2} \partial_{r}\right) v
$$


The function $\underline{v}$ is its solutions as $\lambda^{2}=\underline{\lambda}^{2}=\mu$. Substituting the right hand side of this identity into the integral instead of the expression $r^{-1} \Delta_{\theta, \varphi} \underline{v}$ for $\lambda^{2}=\underline{\lambda}^{2}, v=\underline{v}, r=t$, we obtain

$$
\mathrm{K} \underline{w}=-i r^{-1} \int_{0}^{r} e^{i \underline{\lambda}(r-t)}\left(\underline{\lambda}^{2} t \underline{v}+\frac{1}{t} \partial_{t}\left(t^{2} \partial_{t} \underline{v}\right)\right) d t .
$$

Integrating by parts and taking into consideration the relation $v(0)=0$, we obtain the right hand side of the second identity in 32 . The proof is complete.

3.2. $\quad$ Formulae for solutions. Substituting the expressions $\lambda_{\kappa}^{ \pm}= \pm \lambda_{n, m}$ and $v_{\kappa}$ from (31) into the fraction $v / r$ and the integral (34) (instead of $\underline{\lambda}$ and $\underline{v}$ ), as well as $d=0$, we obtain the explicit formulas for the eigenfunctions of the problem. The following theorem holds true.

Theorem 2. Nonzero eigenvalues $\lambda_{n, m}^{ \pm}$of Problem 1 are equal to $\pm \lambda_{n, m}$, where $\lambda_{n, m}=\rho_{n, m} R^{-1}, R$ is the radius of the ball, and the number $\rho_{n, m}$ are zeroes of the functions $\psi_{n}(z), m, n \in \mathbb{N}$. The components $u_{r}$ and $w=u_{\varphi}+i u_{\theta}$ of the eigenfunctions $u_{\kappa}^{ \pm}$of Problem 1 in the spherical coordinates are calculated by the formulae,

$$
\begin{gathered}
\left(u_{r}\right)_{\kappa}^{ \pm}=c_{\kappa}^{ \pm}\left(\lambda_{n, m}^{ \pm} r\right)^{-1} \psi_{n}\left(\lambda_{n, m}^{ \pm} r\right) Y_{n}^{k}(\theta, \varphi), \\
\left(u_{\varphi}+i u_{\theta}\right)_{\kappa}^{ \pm}=c_{\kappa}^{ \pm}\left(\lambda_{n, m}^{ \pm} r\right)^{-1} \Phi_{n}\left(\lambda_{n, m}^{ \pm} r\right) \mathrm{H} Y_{n}^{k}(\theta, \varphi),
\end{gathered}
$$

where $i$ is the imaginary unit, $c_{\kappa}^{ \pm} \in \mathbb{R}, m, n \in \mathbb{N},|k| \leq n, \kappa=(n, m, k)$,

$$
\begin{gathered}
\Phi_{n}\left(\lambda_{n, m}^{ \pm} r\right)=\int_{0}^{r} e^{i \lambda_{n, m}^{ \pm}(r-t)} \psi_{n}\left(\lambda_{n, m}^{ \pm} t\right) t^{-1} d t \\
\mathrm{H} Y_{n}^{k}(\theta, \varphi)=\left(\sin ^{-1} \theta \partial_{\varphi}+i \partial_{\theta}\right) Y_{n}^{k}(\theta, \varphi) .
\end{gathered}
$$

The functions $u_{r}, u_{\theta}, u_{\varphi}$ are infinitely differentiable everywhere in $\bar{B}$ except the axis $x_{3}$, on which $r \sin \theta=0$, and they are bounded in $\bar{B}$. In terms of the initial coordinates $x_{1}, x_{2}, x_{2}$ the components $u_{j}$ of the eigenfunctions of Problem 1 belong to $C^{\infty}(\bar{B})$.

Proof. In terms of the functions $u_{r}$ and $w=u_{\varphi}+i u_{\theta}$, they are expressed as follows,

$$
u_{1}=u_{r} Y_{1}^{1}+\operatorname{Re}\left(w \overline{\mathrm{H}} Y_{1}^{1}\right), \quad u_{2}=u_{r} Y_{1}^{-1}+\operatorname{Re}\left(w \overline{\mathrm{H}} Y_{1}^{-1}\right), \quad u_{3}=u_{r} Y_{1}^{0}+\operatorname{Re}\left(w \overline{\mathrm{H}} Y_{1}^{0}\right),
$$

where in accordance with the textbook by Vladimirov [3]

$$
\begin{gathered}
x_{1} / r=Y_{1}^{1}(\theta, \varphi)=\sin \theta \cos \varphi, \quad x_{2} / r=Y_{1}^{-1}(\theta, \varphi)=\sin \theta \sin \varphi, \quad x_{3} / r=Y_{1}^{0}(\theta)=\cos \theta, \\
H Y_{1}^{1}=-\sin \varphi+i \cos \theta \cos \varphi, \quad H Y_{1}^{-1}=\cos \varphi+i \cos \theta \sin \varphi, \quad H Y_{1}^{0}=-i \sin \theta .
\end{gathered}
$$

The smoothness of the vector functions $u_{\kappa}^{ \pm}(x)$ in $\bar{B}$ follows from the general theory (see statement b) in Subsection 1.4) and can be checked by straightforward calculations. The proof is complete.

We represent the vector functions $u_{\kappa}^{ \pm}$as a sum of three real orthogonal vectors. Employing the frame $\mathbf{i}_{r}, \mathbf{i}_{\theta}, \mathbf{i}_{\varphi}$ and splitting real and imaginary parts in (37), (38), (39), we have

$$
\begin{gathered}
u_{\kappa}^{ \pm}=c_{\kappa}^{ \pm}\left(\lambda_{n, m}^{ \pm} r\right)^{-1} \psi_{n}\left(\lambda_{n, m}^{ \pm} r\right) Y_{n}^{k}(\theta, \varphi) \mathbf{i}_{r}+ \\
c_{\kappa}^{ \pm}\left(\lambda_{n, m}^{ \pm} r\right)^{-1} \operatorname{Re}\left[\Phi_{n}\left(\lambda_{n, m}^{ \pm} r\right)\right]\left(\operatorname{Re} H Y_{n}^{k} \mathbf{i}_{\varphi}+\operatorname{Im} H Y_{n}^{k} \mathbf{i}_{\theta}\right)+ \\
c_{\kappa}^{ \pm}\left(\lambda_{n, m}^{ \pm} r\right)^{-1} \operatorname{Im}\left[\Phi_{n}\left(\lambda_{n, m}^{ \pm} r\right)\right]\left(-\operatorname{Im} H Y_{n}^{k} \mathbf{i}_{\varphi}+\operatorname{Re} H Y_{n}^{k} \mathbf{i}_{\theta}\right) .
\end{gathered}
$$

These formulae allow us to represent the motion of liquid vortical flow in the ball whose speed is $u_{\kappa}^{ \pm}(x)$ as $n=1,2, \ldots$. The vorticity of these flows rot $u_{\kappa}^{ \pm}$equalling $\lambda_{n, m}^{ \pm} u_{\kappa}^{ \pm}$is nonzero at each point of the ball. 
3.3. The property of functions $\Phi_{n}\left(\lambda_{n, m}^{ \pm} r\right)$. The functions $\psi_{n}\left(\lambda_{n, m}^{ \pm} r\right), Y_{n}^{k}(\theta, \varphi)$ and numbers $\lambda_{n, m}^{ \pm}= \pm \rho_{n, m} / R$ are real. According to (14), $\psi_{n}\left(\lambda_{n, m}^{-} r\right)=(-1)^{n} \psi_{n}\left(\lambda_{n, m} r\right)$. This is why

$$
\begin{gathered}
\Phi_{n}\left(\lambda_{n, m}^{-} r\right)=\int_{0}^{r} e^{-i \lambda_{n, m}(r-t)} \psi_{n}\left(-\lambda_{n, m} t\right) t^{-1} d t= \\
=(-1)^{n} \int_{0}^{r} e^{-i \lambda_{n, m}(r-t)} \psi_{n}\left(\lambda_{n, m} t\right) t^{-1} d t=(-1)^{n} \overline{\Phi_{n}\left(\lambda_{n, m} r\right)} .
\end{gathered}
$$

Let us prove that the number $\Phi_{n}\left(\lambda_{n, m} R\right)$ is real and thus

$$
\Phi_{n}\left(\rho_{n, m}\right)=\int_{0}^{R} \cos \lambda_{n, m}(R-t) \psi_{n}\left(\lambda_{n, m} t\right) t^{-1} d t .
$$

By the construction, the vector functions $u_{\kappa}^{ \pm}(x)$ satisfy equation (1) as $\lambda= \pm \lambda_{n, m}$, and the complex functions

$$
w_{\kappa}^{ \pm}=\left(u_{\varphi}+i u_{\theta}\right)_{\kappa}^{ \pm}=a_{\kappa}^{ \pm}\left(\lambda_{n, m}^{ \pm} r\right)^{-1} \Phi_{n}\left(\lambda_{n, m}^{ \pm} r\right) H Y_{n}^{k}(\theta, \varphi), \quad a_{\kappa}^{ \pm} \in \mathbb{R}
$$

satisfy system of equations (32) as $\lambda= \pm \lambda_{n, m}, v=v_{\kappa}(x)$ and $\left.v_{\kappa}\right|_{r=R}=0$.

By the second equation in (32) we see that as $r \rightarrow R$,

$$
\left.\operatorname{Re} K w_{\kappa}^{ \pm}\right|_{r \rightarrow R}= \pm\left.\lambda_{n, m} v_{\kappa}\right|_{r=R}=0 .
$$

The composition $\mathrm{KH}$ of the operators $\mathrm{K}$ and $\mathrm{H}$ on the real functions $Y_{n}^{k}(\theta, \varphi)$ reads as

$$
\begin{gathered}
\mathrm{KH} Y_{n}^{k}=\sin ^{-1} \theta\left(\partial_{\theta} \sin \theta+i \partial_{\varphi}\right)\left(\sin ^{-1} \theta \partial_{\varphi}+i \partial_{\theta}\right) Y_{n}^{k}= \\
\sin ^{-1}\left(\partial_{\theta} \partial_{\varphi}-\partial_{\varphi} \partial_{\theta}\right) Y_{n}^{k}+i \Delta_{\theta, \varphi} Y_{n}^{k}=i n(n+1) Y_{n}^{k}
\end{gathered}
$$

Hence,

$$
\left.\operatorname{Re} \mathrm{K} w_{\kappa}^{ \pm}\right|_{r=R}=-n(n+1) a_{\kappa}^{ \pm}\left(\rho_{n, m}^{ \pm}\right)^{-1} \operatorname{Im} \Phi_{n}\left(\rho_{n, m}^{ \pm}\right) Y_{n}^{k}(\theta, \varphi)=0
$$

for each $\theta$ and $\varphi$. Therefore, $\operatorname{Im} \Phi_{n}\left(\rho_{n, m}\right)=0$ and the number $\Phi_{n}\left(\rho_{n, m}\right)$ is real.

\section{Solving of SPeCtral Problem 1 as $\lambda=0$}

4.1. Reduction of Problem 1 as $\lambda=0$ to Neumann spectral problem. We shall seek the vector eigenfunctions of the curl operator associated with the zero eigenvalue among the solutions of the following spectral problem.

Problem 3. To find nonzero eigenvalues $\mu$ and vector eigenfunctions $\mathbf{u}(\mathbf{x})$ in $\mathbf{L}_{2}(G)$ of the gradient-divergence operator such that

$$
-\nabla \operatorname{div} \mathbf{u}=\mu \mathbf{u} \quad \text { in } \quad G,\left.\quad \mathbf{n} \cdot \mathbf{u}\right|_{\Gamma}=0,
$$

where $\mathbf{n} \cdot \mathbf{u}$ is the projection of the vector $\mathbf{u}$ on the normal vector $\mathbf{n}$.

As the domain $\mathcal{M}_{\mathcal{G D}}$ of the operator $\mathcal{G D}$ in Problem 3, we take the vector functions $\mathbf{u}(\mathbf{x})$ in $\mathcal{C}^{2}(G) \cap \mathcal{C}^{1}(\bar{G})$ satisfying the boundary condition $\left.\mathbf{n} \cdot \mathbf{u}\right|_{\Gamma}=0$ and the assumption $\nabla \operatorname{div} \mathbf{u} \in \mathbf{L}_{2}(G)$.

The problem is related to the Neumann spectral problem for the scalar Laplace operator.

Problem 4. To find all the eigenvalues $\nu$ and the eigenfunctions $g(\mathbf{x})$ of the Laplace operator $-\Delta$ such that

$$
-\Delta g=\nu g \quad \text { in } G,\left.\quad \mathbf{n} \cdot \nabla g\right|_{\Gamma}=0 .
$$

As the domain $\mathcal{M}_{\mathcal{N}}$ of the operator $\mathcal{N}$ in Problem 4, we take the functions $g(\mathbf{x})$ in $\mathcal{C}^{2}(G) \cap$ $\mathcal{C}^{1}(\bar{G})$ satisfying the conditions $\left.\mathbf{n} \cdot \nabla g\right|_{\Gamma}=0, \Delta g \in L_{2}(G)$.

It is easy to make sure that following lemma holds true.

Lemma 3. Each solution $(\mu, \mathbf{u})$ of Problem 3 in the domain $G$ corresponds to the solution $(\nu, g)=(\mu, \operatorname{div} \mathbf{u})$ of Problem 4. And vice versa, each solution $(\nu, g)$ of Problem 4 corresponds to the solution $(\mu, \mathbf{u})=(\nu, \nabla g)$ of Problem 3 . 
4.2. Solving of spectral problem 4 in ball. The solution to this problem is known. In accordance with the book by V.S. Vladimirov [3,

the eigenvalues of the operator $-\Delta$ in a ball $B$ subject to the Neumann condition are equal to $\nu_{n, m}^{2}$, where $\nu_{n, m}=\alpha_{n, m} R^{-1}, n \geq 0, m \in N$, and the numbers $\alpha_{n, m}>0$ are the zeroes of the derivatives $\psi_{n}^{\prime}(z)$ of the functions $\psi_{n}(z)$, i.e., $\psi_{n}^{\prime}\left(\alpha_{n, m}\right)=0$. The eigenfunctions $g_{\kappa}$ associated with $\nu_{n, m}^{2}$ read as follows,

$$
g_{\kappa}(r, \theta, \varphi)=c_{\kappa} \psi_{n}\left(\alpha_{n, m} r / R\right) Y_{n}^{k}(\theta, \varphi),
$$

where $\kappa=(n, m, k)$ is a multi-index, $c_{\kappa}$ are arbitrary real constants, $Y_{n}^{k}(\theta, \varphi)$ are real spherical functions, $n \geq 0,|k| \leq n, m \in N$.

The functions $g_{\kappa}(x)$ belong to the class $C^{\infty}(\bar{B})$ and for different $\kappa$, they are orthogonal in $L_{2}(B)$. The system of the functions $\left\{g_{\kappa}\right\}$ is complete in $L_{2}(B)$ [5]. Normalizing them, we obtain a orthonormalized basis in $L_{2}(B)$.

4.3. Solving of spectral problem 3 in ball. According to Lemma 3, the vector functions $\mathbf{q}_{\kappa}(x)=\nabla g_{\kappa}(x)$ solve Problem 3 for $\mu_{n, m}=\alpha_{n, m}^{2} R^{-2}$ in $\mathbf{L}_{2}(B)$. Their components $\left(q_{r}, q_{\theta}, q_{\varphi}\right)$ read as follows,

$$
\begin{gathered}
q_{r, \kappa}(r, \theta, \varphi)=c_{\kappa}\left(\alpha_{n, m} / R\right) \psi_{n}^{\prime}\left(\alpha_{n, m} r / R\right) Y_{n}^{k}(\theta, \varphi), \\
\left(q_{\varphi}+i q_{\theta}\right)_{\kappa}=c_{\kappa}(1 / r) \psi_{n}\left(\alpha_{n, m} r / R\right) \mathrm{H} Y_{n}^{k}(\theta, \varphi) .
\end{gathered}
$$

As $\kappa=(0, m, 0)$, we have $Y_{0}^{0}(\theta, \varphi)=1, \mathrm{H} Y_{0}^{0}=0$. Hence,

$$
\begin{aligned}
& q_{r,(0, m, 0)}(r)=c_{(0, m, 0)}\left(\alpha_{0, m} / R\right) \psi_{0}^{\prime}\left(\alpha_{0, m} r / R\right), \\
& \left(q_{\varphi}+i q_{\theta}\right)_{(0, m, 0)}=0 .
\end{aligned}
$$

By these formulae, one can easily write the normalizing constants $c_{\kappa}$ guaranteeing $\left\|\mathbf{q}_{\kappa}\right\|=1$.

4.4. Solving of spectral problem 1 as $\lambda=0$ in ball. The numbers $\mu_{n, m}=\alpha_{n, m}^{2} R^{-2}$ are positive for each $n \geq 0, m \in N$. This is why the vector functions $\mathbf{q}_{\kappa}$ solve also Problem 1 as $\lambda=0$. Moreover, $\mathbf{q}_{\kappa}$ and $\mathbf{q}_{\kappa^{\prime}}$ are orthogonal as $\kappa^{\prime} \neq \kappa$.

Indeed, by Gauss-Ostrogradsky formula,

$$
\int_{B} \nabla g_{\kappa^{\prime}} \cdot \nabla g_{\kappa} d x=-\int_{B} g_{\kappa^{\prime}} \Delta g_{\kappa} d x+\int_{S} g_{\kappa^{\prime}}(n \cdot \nabla) g_{\kappa} d S .
$$

The functions $g_{\kappa}(x)$ solve Problem 4, they satisfy Helmholtz equation (51) as $\nu=\alpha_{n, m}^{2} / R^{2}>0$ subject to the Neumann condition. Hence, the boundary integral disappears and

$$
\int_{B} \mathbf{q}_{\kappa^{\prime}} \cdot \mathbf{q}_{\kappa} d x=\frac{\alpha_{n, m}^{2}}{R^{2}} \int_{B} g_{\kappa^{\prime}} g_{\kappa} d x .
$$

But according to (52), the functions $g_{\kappa}(x)$ and $g_{\kappa^{\prime}}(x)$ are orthogonal in $L_{2}(B)$ as $\kappa^{\prime} \neq \kappa$. Hence, the last integral in (56) vanishes and the vector functions $\mathbf{q}_{\kappa}$ and $\mathbf{q}_{\kappa^{\prime}}$ are orthogonal in $\mathbf{L}_{2}(B)$.

Observe that $\left\|\mathbf{q}_{\kappa}(x)\right\|=\left(\alpha_{n, m} / R\right)\left\|g_{\kappa}(x)\right\|$.

\section{SPACE $\mathbf{L}_{2}(B)$ AND EIGENFUnCtions FOR CURL OPERATOR}

5.1. Subspace $\mathcal{A}=\nabla H^{1}(B)$. We denote by $\mathcal{A}$ the linear subspace in $\mathbf{L}_{2}(B)$ formed by an orthonormalized system of the vector-functions $\left\{\mathbf{q}_{\kappa}(x)\right\}$. In fact,

$$
\mathcal{A}=\left\{\nabla h: \quad h \in H^{1}(B)\right\} .
$$

Indeed, for each element, $\mathbf{q}_{\kappa}(x)=\nabla g_{\kappa}$, where $g_{\kappa} \in H^{1}(B)$. On the other hand, a function $h$ in $H^{1}(B)$ can be expanded into the convergent series

$$
h=\sum_{\kappa}\left(h, \widehat{g}_{\kappa}\right) \widehat{g}_{\kappa}, \quad \widehat{g}_{\kappa}=\left(\alpha_{n, m} / R\right) g_{\kappa}, \quad\left(\widehat{g}_{\kappa}, \widehat{g}_{\kappa^{\prime}}\right)=\delta_{\kappa, \kappa^{\prime}}
$$


5.2. Subspace $\mathcal{B}=\mathbf{V}^{0}(B)$. We denote by $\mathbf{q}_{\kappa}^{ \pm}(x)$ the solutions to Problem 1 associated in accordance with Theorem 2 with the eigenvalues $\lambda_{n, m}^{ \pm}, n, m \in \mathbb{N}$, and normalized in $\mathbf{L}_{2}(B)$, i.e., $\left\|\mathbf{q}_{\kappa}^{ \pm}(x)\right\|=1$. They belong to the subspace

$$
\mathbf{V}^{0}(B)=\left\{\mathbf{u} \in \mathbf{L}_{2}(B): \operatorname{div} \mathbf{u}=0,\left.\quad \mathbf{n} \cdot \mathbf{u}\right|_{S}=0, \quad\|\mathbf{u}\|_{\mathbf{V}^{0}(B)}=\|\mathbf{u}\|_{\mathbf{L}_{2}(B)}\right\},
$$

where $\operatorname{div} \mathbf{u}=0,\left.\mathbf{n} \cdot \mathbf{u}\right|_{S}=0$ are treated in the sense of the distributions theory,

$$
\mathbf{V}^{0}(B)=\left\{\mathbf{u} \in \mathbf{L}_{2}(B): \int_{B} \mathbf{u} \cdot \nabla h d x=0, \quad \text { for each } h \in H^{1}(B)\right\} .
$$

It is obvious that $\mathcal{A}$ and $\mathcal{B} \equiv \mathbf{V}^{0}(B)$ are orthogonal subspaces in $\mathbf{L}_{2}(B)$. By $\mathcal{B}^{ \pm}$we indicate the subspaces in $\mathcal{B}$ formed by the systems of the vector functions $\left\{\mathbf{q}_{\kappa}^{ \pm}(x)\right\}$. The following lemma holds true.

Lemma 4. The vector functions $\mathbf{q}_{\kappa}^{+}(x)$ (respectively, $\mathbf{q}_{\kappa}^{-}(x)$ ) are orthogonal for different $\kappa$. The vector functions $\mathbf{q}_{\kappa}^{+}(x)$ and $\mathbf{q}_{\kappa}^{-}(x)$ are orthogonal for all $\kappa$.

Proof. We employ the Green formula for the curl operator,

$$
\int_{B} \operatorname{rot} \mathbf{u} \cdot \mathbf{v} d \mathbf{x}-\int_{B} \mathbf{u} \cdot \operatorname{rot} \mathbf{v} d \mathbf{x}=\int_{S}[\mathbf{u}, \mathbf{v}] \cdot \mathbf{n} d S .
$$

On the sphere $S$, the scalar triple product $[\mathbf{u}, \mathbf{v}] \cdot \mathbf{n}$ coincides with the determinant

$$
\left|\begin{array}{ccc}
1 & 0 & 0 \\
u_{r} & u_{\theta} & u_{\varphi} \\
v_{r} & v_{\theta} & v_{\varphi}
\end{array}\right|
$$

and equals $u_{\theta} v_{\varphi}-u_{\varphi} v_{\theta}$ or $\operatorname{Im}(W \bar{V})$ in the complex notations $W=\left(u_{\varphi}+i u_{\theta}\right)$ and $\bar{V}=\left(v_{\varphi}-i v_{\theta}\right)$.

Let us prove the orthogonality of the vector functions $\mathbf{q}_{\kappa^{\prime}}^{+}(x)$ and $\mathbf{q}_{\kappa}^{+}(x)$ as $\kappa^{\prime} \neq \kappa$. They solve Problem 1 and are calculated by the formulae (36), (37), where $\lambda_{n, m}^{+}=\rho_{n, m} / R$ and $c_{\kappa}^{+}$ are real constants.

We first consider the case $\left(n^{\prime}, m^{\prime}\right) \neq(n, m)$, and hence, $\lambda_{n^{\prime}, m^{\prime}}^{+} \neq \lambda_{n, m}^{+}$. Substituting these functions into formula (61), we obtain the identity

$$
\left(\lambda_{n^{\prime}, m^{\prime}}-\lambda_{n, m}\right) \int_{B} \mathbf{q}_{\kappa^{\prime}}^{+} \cdot \mathbf{q}_{\kappa}^{+} d x=\operatorname{Im} \int_{0}^{\pi} \int_{0}^{2 \pi} W_{k^{\prime}}^{+} \bar{W}_{k}^{+} \sin \theta d \theta d \varphi .
$$

The orthogonality will be proven if the latter integral $I$ vanishes. In accordance with formulae (37), it read as

$$
I=A \operatorname{Im} \int_{0}^{\pi} \int_{0}^{2 \pi} \mathrm{H} Y_{n^{\prime}}^{k^{\prime}}(\theta, \varphi) \overline{\mathrm{H}} Y_{n}^{k}(\theta, \varphi) \sin \theta d \theta d \varphi,
$$

where $A=c_{\kappa^{\prime}}^{+}\left(\rho_{n^{\prime}, m^{\prime}}\right)^{-1} c_{\kappa}^{+}\left(\rho_{n, m}\right)^{-1} \Phi_{n^{\prime}}\left(\rho_{n^{\prime}, m^{\prime}}\right) \bar{\Phi}_{n}\left(\rho_{n, m}\right)$ is a real constant according to Subsection 3.3 .

We move the operator $\mathrm{H}$ in this integral by integration by parts. We have

$$
\begin{gathered}
\operatorname{Im}\left[A \int_{0}^{\pi} \int_{0}^{2 \pi} Y_{n^{\prime}}^{k^{\prime}}(\theta, \varphi)\left[-\sin ^{-1} \theta \partial_{\theta}\left(\sin \theta \partial_{\theta}\right)-\sin ^{-2} \theta \partial_{\varphi}^{2}\right] Y_{n}^{k}(\theta, \varphi) \sin \theta d \theta d \varphi\right]+ \\
\operatorname{Im}\left[i A \int_{0}^{\pi} \int_{0}^{2 \pi} Y_{n^{\prime}}^{k^{\prime}}(\theta, \varphi)\left[\sin ^{-1} \theta\left(\partial_{\varphi} \partial_{\theta}-\partial_{\theta} \partial_{\varphi}\right)\right] Y_{n}^{k}(\theta, \varphi) \sin \theta d \theta d \varphi\right] .
\end{gathered}
$$

The latter integral vanishes, since the spherical functions and all their derivatives w.r.t. $\varphi$ and $\theta$ are continuous. The operator in the brackets in the first integral is the Laplace-Beltrami 
operator, $-\Delta_{\theta \varphi}$. According to the property of the spherical functions, $-\Delta_{\theta \varphi} Y_{n}^{k}(\theta, \varphi)=n(n+$ 1) $Y_{n}^{k}(\theta, k)$. Substituting this expression into the integral, we get

$$
\left(\lambda_{n^{\prime}, m^{\prime}}-\lambda_{n, m}\right) \int_{B} q_{\kappa^{\prime}}^{+} \cdot q_{\kappa}^{+} d x=\operatorname{Im}\left[n(n+1) A \int_{0}^{\pi} \int_{0}^{2 \pi} Y_{n^{\prime}}^{k^{\prime}} Y_{n}^{k} \sin \theta d \theta d \varphi\right] .
$$

Since the spherical functions are orthogonal as $\left(n^{\prime}, k^{\prime}\right) \neq(n, k)$, this integral vanishes. Hence, the vector functions $\mathbf{q}_{\kappa^{\prime}}^{+}(x)$ and $\mathbf{q}_{\kappa}^{+}(x)$ are orthogonal as $\left(n^{\prime}, m^{\prime}\right) \neq(n, m)$ and $\left(n^{\prime}, k^{\prime}\right) \neq(n, k)$.

If $\left(n^{\prime}, k^{\prime}\right)=(n, k), m^{\prime} \neq m$, the integral in the right hand side of (65) is a real number. The numbers $c_{\kappa}, \Phi_{n}\left(\rho_{n, m}\right)$, and $A$ are real as well, and hence $\mathbf{q}_{k, m^{\prime}, n}^{+}(x)$ and $\mathbf{q}_{k, m, n}^{+}(x)$ are orthogonal.

In the case $\left(n^{\prime}, m^{\prime}\right)=(n, m)$ and $k^{\prime} \neq k$, formula (65) is not suitable, since both its sides vanishes. According to formulae (36), (37), we have

$$
\begin{gathered}
\int_{B} \mathbf{q}_{k^{\prime}, m, n}^{+} \cdot \mathbf{q}_{k, m, n}^{+} d x=c_{k^{\prime}, m, n}^{+} c_{k^{\prime}, m, n}^{+} \lambda_{m, n}^{-2}\left[\int_{0}^{R} \psi_{n}^{2}\left(\lambda_{n, m} r\right) d r \int_{0}^{\pi} \int_{0}^{2 \pi} Y_{n}^{k^{\prime}}(\theta, \varphi) Y_{n}^{k}(\theta, \varphi) \sin \theta d \theta d \varphi+\right. \\
\left.+\int_{0}^{R} \Phi_{n}\left(\lambda_{n, m} r\right) \bar{\Phi}_{n}\left(\lambda_{n, m} r\right) d r \int_{0}^{\pi} \int_{0}^{2 \pi} \mathrm{H} Y_{n}^{k^{\prime}}(\theta, \varphi) \overline{\mathrm{H}} Y_{n}^{k}(\theta, \varphi) \sin \theta d \theta\right] .
\end{gathered}
$$

Because of the orthogonality of the functions $Y_{n}^{k^{\prime}}$ and $Y_{n}^{k}$ in $L_{2}\left(S_{1}\right)$ both integrals disappear and the vectors $\mathbf{q}_{k^{\prime}, m, n}^{+}$and $\mathbf{q}_{k, m, n}^{+}$are thus orthogonal.

The orthogonality of the vector functions $\mathbf{q}_{\kappa^{\prime}}^{-}(x)$ and $\mathbf{q}_{\kappa}^{-}(x)$ as $\kappa^{\prime} \neq \kappa$ can be proven in the same way.

For all $\kappa^{\prime}$ and $\kappa$, consider the eigenfunctions $\mathbf{q}_{\kappa^{\prime}}^{+}(x)$ and $\mathbf{q}_{\kappa}^{-}(x)$ associated with the eigenvalues $\lambda_{n, m}$ and $-\lambda_{n, m}$ of opposite signs. Reproducing the above calculations, we have

$$
\begin{gathered}
\left(\lambda_{n^{\prime}, m^{\prime}}+\lambda_{n, m}\right) \int_{B} \mathbf{q}_{\kappa^{\prime}}^{+} \cdot \mathbf{q}_{\kappa}^{-} d x=\operatorname{Im} \int_{0}^{\pi} \int_{0}^{2 \pi} W_{k^{\prime}}^{+} \bar{W}_{k}^{-} \sin \theta d \theta d \varphi= \\
=\operatorname{Im}\left[n(n+1) B \int_{0}^{\pi} \int_{0}^{2 \pi} Y_{n^{\prime}}^{k^{\prime}}(\theta, \varphi) Y_{n}^{k}(\theta, \varphi) \sin \theta d \theta d \varphi\right],
\end{gathered}
$$

where the constant $B=(-1)^{(n+1)} c_{\kappa^{\prime}}^{+}\left(\rho_{n^{\prime}, m^{\prime}}\right)^{-1} c_{\kappa}^{-}\left(\rho_{n, m}\right)^{-1} \Phi_{n^{\prime}}\left(\rho_{n^{\prime}, m^{\prime}}\right) \Phi_{n}\left(\rho_{n, m}\right)$ is real.

The right hand side in (67) disappears for all $\kappa^{\prime}$ and $\kappa$. Therefore, the vector functions $\mathbf{q}_{\kappa^{\prime}}^{+}(x)$ and $\mathbf{q}_{\kappa}^{-}(x)$ are orthogonal. The proof is complete.

5.3. H. Weil expansion. The completeness of the eigenfunctions for the Laplace operators subject to the Dirichlet and Neumann condition in $L_{2}(B)$ implies that the system of the vector functions $\left\{\mathbf{q}_{\kappa}(x)\right\}$ is complete in the subspace $\mathcal{A}$, the union of the systems $\left\{\mathbf{q}_{\kappa}^{+}(x)\right\}$ and $\left\{\mathbf{q}_{\kappa}^{-}(x)\right\}$ is complete in the subspace $\mathcal{B}$. There are no other solutions to Problem 1.

The subspaces $\mathcal{A}$ and $\mathcal{B}$ are orthogonal in $\mathbf{L}_{2}(B)$. In the case of a ball, their union coincides with $\mathbf{L}_{2}(B)$ (see H. Weil [10]). Hence, we obtain an orthogonal decomposition of the space $\mathbf{L}_{2}(B)$ in terms of the vector eigenfunctions of the curl operator

$$
\mathbf{L}_{2}(B)=\mathcal{A} \oplus \mathcal{B}=\mathcal{A} \oplus \mathcal{B}^{+} \oplus \mathcal{B}^{-}
$$

Theorem 3. The union of the systems $\left\{\mathbf{q}_{\kappa}(x)\right\},\left\{\mathbf{q}_{\kappa}^{+}(x)\right\}$ and $\left\{\mathbf{q}_{\kappa}^{-}(x)\right\}$ of the vector eigenfunctions for Problem 1 makes an orthonormalized basis in the space $\mathbf{L}_{2}(B)$. Each vector function in $\mathbf{L}_{2}(B)$ can be expanded into the Fourier series in terms of this basis.

The Weil expansion of a vector field $\mathbf{f}$ in $\mathbf{L}_{2}(B)$ into a curlfree field $\mathbf{a}$ and a solenoidal field $\mathbf{b}$ reads as $\mathbf{f}(\mathbf{x})=\mathbf{a}(\mathbf{x})+\mathbf{b}(\mathbf{x})$, where

$$
\mathbf{a}=\sum_{n=0}^{\infty} \sum_{m=1}^{\infty} \sum_{k=-n}^{n}\left(\mathbf{f}, \mathbf{q}_{n, m, k}\right) \mathbf{q}_{n, m, k}(\mathbf{x}),
$$




$$
\mathbf{b}=\sum_{n=1}^{\infty} \sum_{m=1}^{\infty} \sum_{k=-n}^{n}\left[\left(\mathbf{f}, \mathbf{q}_{n, m, k}^{+}\right) \mathbf{q}_{n, m, k}^{+}(\mathbf{x})+\left(\mathbf{f}, \mathbf{q}_{n, m, k}^{-}\right) \mathbf{q}_{n, m, k}^{-}(\mathbf{x})\right]
$$

the summation of series (69), (70) is in $n, m$ satisfying $0<\alpha_{n, m}<N$ and $0<\rho_{n, m}<N$, where $N \rightarrow \infty$.

The Parseval-Steklov identity $\|\mathbf{f}\|^{2}=\|\mathbf{a}\|^{2}+\|\mathbf{b}\|^{2}$ holds true and we write it as

$$
\|\mathbf{f}\|^{2}=\sum_{N=1}^{\infty} \sum_{(n, m) \in \mathbb{P}_{N}} \sum_{k \in[-n, n]}\left[\left(\mathbf{f}, \mathbf{q}_{n, m, k}\right)^{2}+\left(\mathbf{f}, \mathbf{q}_{n, m, k}^{+}\right)^{2}+\left(\mathbf{f}, \mathbf{q}_{n, m, k}^{-}\right)^{2}\right]
$$

where the lattice is given by the formula $\mathbb{P}_{N}=\left\{(n, m): 0<\rho_{n, m}<N, 0<\alpha_{n, m}<N\right\}$ and $\mathbf{q}_{0, m, 0}^{ \pm}=0$.

We note that the expansion of the vector field $\mathbf{f}(\mathbf{x})$ into a curlfree field $\nabla h(\mathbf{x})$ and a solenoidal field $\mathbf{u}(\mathbf{x})$ is related with the solution to the Neumann problem

$$
\triangle h=\operatorname{div} \mathbf{f} \quad \text { in } \quad B,\left.\quad \mathbf{n} \cdot \nabla h\right|_{S}=\left.\mathbf{n} \cdot \mathbf{f}\right|_{S},
$$

in the classical or generalized formulation [2].

We obtain the solution to this problem as the series $(69),(70)$. Let us note their properties.

If $\mathbf{f}=\nabla h$, where $h(\mathbf{x})$ is a compactly supported in $B$ infinitely differentiable function, i.e., $h \in \mathcal{D}(B)$, then $\nabla \operatorname{div} \mathbf{f}=\nabla \triangle h$ and for each integer $s>1,(\nabla d i v)^{s} \mathbf{f}=\nabla \triangle^{s} h \in \mathbf{L}_{2}(B)$.

Hence, integrating by parts, we have

$$
\sum_{n=0}^{\infty} \sum_{m=1}^{\infty} \sum_{k=-n}^{n}\left((\nabla d i v)^{s} \mathbf{f}, \mathbf{q}_{n, m, k}\right) \mathbf{q}_{n, m, k}(\mathbf{x})=\sum_{n=0}^{\infty} \sum_{m=1}^{\infty} \sum_{k=-n}^{n}\left(\alpha_{n, m} / R\right)^{2 s}\left(\mathbf{f}, \mathbf{q}_{n, m, k}\right) \mathbf{q}_{n, m, k}(\mathbf{x}) .
$$

The series converges to $(\nabla \operatorname{div})^{s} \mathbf{f}$ in $\mathbf{L}_{2}(B)$ and

$$
\sum_{n=0}^{\infty} \sum_{m=1}^{\infty} \sum_{k=-n}^{n}\left(\alpha_{n, m} / R\right)^{4 s}\left|\left(\mathbf{f}, \mathbf{q}_{n, m, k}\right)\right|^{2}=\left\|(\nabla \operatorname{div})^{s} \mathbf{f}\right\|_{\mathbf{L}_{2}(B)}^{2}
$$

If the vector function $\mathbf{f}$ is solenoidal and its components belong to the space $\mathcal{D}(B)$, then for each integer $s \geq 1,(\text { rot })^{s} \mathbf{f} \in \mathbf{V}^{0}(B)$. Hence, by analogy with above arguments,

$$
\begin{aligned}
& \sum_{n=1}^{\infty} \sum_{m=1}^{\infty} \sum_{k=-n}^{n}\left[\left((\operatorname{rot})^{s} \mathbf{f}, \mathbf{q}_{n, m, k}^{+}\right) \mathbf{q}_{n, m, k}^{+}(\mathbf{x})+\left((\operatorname{rot})^{s} \mathbf{f}, \mathbf{q}_{n, m, k}^{-}\right) \mathbf{q}_{n, m, k}^{-}(\mathbf{x})\right]= \\
= & \sum_{n=1}^{\infty} \sum_{m=1}^{\infty} \sum_{k=-n}^{n}\left(\rho_{n, m} / R\right)^{s}\left[\left(\mathbf{f}, \mathbf{q}_{n, m, k}^{+}\right) \mathbf{q}_{n, m, k}^{+}(\mathbf{x})+(-1)^{s}\left(\mathbf{f}, \mathbf{q}_{n, m, k}^{-}\right) \mathbf{q}_{n, m, k}^{-}(\mathbf{x})\right] .
\end{aligned}
$$

The series converge to $(\mathrm{rot})^{s} \mathbf{f}$ in $\mathbf{L}_{2}(B)$ and

$$
\sum_{n=1}^{\infty} \sum_{m=1}^{\infty} \sum_{k=-n}^{n}\left(\rho_{n, m} / R\right)^{2 s}\left[\left|\left(\mathbf{f}, \mathbf{q}_{n, m, k}^{+}\right)\right|^{2}+\left|\left(\mathbf{f}, \mathbf{q}_{n, m, k}^{-}\right)\right|^{2}\right]=\left\|(\operatorname{rot})^{s} \mathbf{f}\right\|_{\mathbf{L}_{2}(B)}^{2} .
$$

These series converge also in $\mathbf{H}^{l}(B)$ as $l=1,2, \ldots$. Indeed, denote by $\mathbf{S}_{j}$ a partial sum of series (75) and employ estimate (10). We obtain

$$
\left\|\mathbf{S}_{j}-\mathbf{S}_{i}\right\|_{\mathbf{H}^{1}(B)}^{2} \leqslant C\left(\left\|\operatorname{rot}\left(\mathbf{S}_{j}-\mathbf{S}_{i}\right)\right\|_{\mathbf{H}^{0}(B)}^{2}+\left\|\mathbf{S}_{j}-\mathbf{S}_{i}\right\|_{\mathbf{H}^{0}(B)}^{2}\right),
$$

since $\operatorname{div}\left(\mathbf{S}_{j}-\mathbf{S}_{i}\right)=0$ and $\left.\mathbf{n} \cdot\left(\mathbf{S}_{j}-\mathbf{S}_{i}\right)\right|_{S}=0$. As $i, j \rightarrow \infty$, the right hand side in $(77)$ tends to zero in accordance with (76). Hence, the series converges in $\mathbf{H}^{1}(B)$ and so forth. 


\section{Solution of Stokes Spectral Problem}

\subsection{Relation between solutions to spectral problems for Stokes and curl oper-} ators. We pass to the study of the spectral problem for the Stokes operator in a bounded domain $G$ with the viscosity parameter $\nu>0$.

Problem 5. To find all the vector eigenfunctions $(\mathbf{v}(\mathbf{x}), p(\mathbf{x}))$ and the eigenvalues $\mu$ for the Stokes operator such that

$$
\begin{aligned}
-\nu \Delta \mathbf{v}+\nabla p= & \mu \mathbf{v}, \quad \operatorname{div} \mathbf{v}=0 \quad \text { in } \quad G, \\
\left.\mathbf{v}\right|_{\Gamma}=0 . &
\end{aligned}
$$

We note that as an eigenfunction for this operator, one usually regards only the vector function $\mathbf{v}(\mathbf{x})$ since $\nabla p$ can be determined via $v$ and $\mu$. In the monograph 2] by O.A. Ladyzhenskaya, it is shown that in a bounded domain $G$ with a smooth boundary $\Gamma$ this problem has a discrete spectrum $\left\{\mu_{k}\right\}$, where $k=1,2, \ldots$ and each $\mu_{k}$ is of finite multiplicity. We shall specify this result in the case of a ball.

There are useful relations between the solutions to Problems 1 and 5 .

Theorem 4. Let $\mathbf{u}^{+}, \mathbf{u}^{-}$satisfy equations $\operatorname{rot} \mathbf{u}^{ \pm}= \pm \lambda \mathbf{u}^{ \pm}$in the domain $G, \lambda>0$, and $p(\mathbf{x})$ is a harmonic in $G$ function. Then the pair $(\mathbf{v}, p)$, where

$$
\mathbf{v}=\mathbf{u}^{+}+\mathbf{u}^{-}+\nu^{-1} \lambda^{-2} \nabla p
$$

solves Stokes equation (78) for $\mu=\nu \lambda^{2}$.

If the functions $\mathbf{u}^{+}, \mathbf{u}^{-}$, and $p(\mathbf{x})$ satisfy also the boundary conditions

$$
\begin{gathered}
\left.\mathbf{n} \cdot \mathbf{u}^{ \pm}\right|_{\Gamma}=0,\left.\quad\left(\mathbf{u}^{+}+\mathbf{u}^{-}\right)\right|_{\Gamma}=0, \\
\left.(\mathbf{n} \cdot \nabla) p\right|_{\Gamma}=0
\end{gathered}
$$

the solution $(\mathbf{v}, p)$ to Problem 5 for $\mu=\nu \lambda^{2}$ reads as

$$
\mathbf{v}=\mathbf{u}^{+}+\mathbf{u}^{-}, \quad p=\text { Const. }
$$

Proof. The proof of the first statement is based on straightforward calculations taking into consideration that the functions $\mathbf{u}^{+}$and $\mathbf{u}^{-}$solve equations (6), (8). Indeed,

$$
-\nu \Delta \mathbf{v}+\nabla p=\nu \lambda^{2}\left(\mathbf{u}^{+}+\mathbf{u}^{-}\right)+\nabla p=\nu \lambda^{2} \mathbf{v} .
$$

If $p$ satisfies homogeneous Neumann condition (82), then $p=$ Const. Homogeneous Neumann problem $(82)$ for the function $p(\mathbf{x})$ harmonic in the bounded domain $G$ with the smooth boundary $\Gamma$ has the solution $p=$ Const, since it follows from the Gauss-Ostrogradsky formula that

$$
\int_{\mathbf{G}}|\nabla p|^{2} d x=0 .
$$

Hence, expansion $(80)$ for the vector $\mathbf{v}$ is simplified and casts into the form $\mathbf{v}=\mathbf{u}^{+}+\mathbf{u}^{-}$, while the boundary condition $\left.\mathbf{v}\right|_{\Gamma}=0$ follows from the relation $\left.\quad\left(\mathbf{u}^{+}+\mathbf{u}^{-}\right)\right|_{\Gamma}=0$.

On the other hand, the following theorem holds true.

Theorem 5. a) Suppose a vector function $(\mathbf{v}(\mathbf{x}), p(\mathbf{x}))$ solves Stokes equation (78) for $\mu>0$, $\mathbf{v}(\mathbf{x}) \neq 0, p(\mathbf{x})$ is a harmonic in $G$ function and $\lambda=\sqrt{\mu \nu^{-1}}$. Then the vector function $\mathbf{v}$ can be represented as the sum

$$
\mathbf{v}=\mathbf{w}+\mu^{-1} \nabla p
$$

where $\mathbf{w}$ satisfies the equation

$$
(\operatorname{rot}+\lambda I)(\operatorname{rot}-\lambda I) \mathbf{w}=0, \quad \operatorname{div} \mathbf{w}=0 .
$$

b) If $p(\mathbf{x})$ satisfies boundary condition 82 , then $\nabla p(\mathbf{x})=0$ and $\mathbf{v}=\mathbf{w}$. 
In the case $G=B$, there exist vector functions $\mathbf{u}^{ \pm}$solving equations $\operatorname{rot} \mathbf{u}^{ \pm}= \pm \lambda \mathbf{u}^{ \pm}$and satisfying boundary conditions (81) such that the vector function $\mathbf{v}$ is represented as the sum

$$
\mathbf{v}=\mathbf{u}^{+}+\mathbf{u}^{-} \text {. }
$$

Proof. The vector functions $\mathbf{v}(\mathbf{x})$ and $\nabla p(\mathbf{x})$ satisfy equations (78). We write first three of them as

$$
(\operatorname{rot}+\lambda \mathrm{I})(\operatorname{rot}-\lambda \mathrm{I}) \mathbf{v}=-\nu^{-1} \nabla p .
$$

Fixing $p$, we consider relation $(88)$ as a matrix differential equation for the vector $\mathbf{v}$. Since $\operatorname{rot} \nabla p \equiv 0$ and $\mu=\nu \lambda^{2}$, then $\mu^{-1} \nabla p$ is its particular solutions and the expression $\mathbf{w}=$ $\mathbf{v}-\mu^{-1} \nabla p$ solves homogeneous equation, i.e., the first equation in (86). The second equation, $\operatorname{div} \mathbf{w}=0$, follows from the equation $\operatorname{div} \mathbf{v}=0$.

Moreover, $\left.\mathbf{n} \cdot \mathbf{w}\right|_{\Gamma}=\left.\mathbf{n} \cdot \mathbf{v}\right|_{\Gamma}-\left.\mu^{-1} \mathbf{n} \cdot \nabla p\right|_{\Gamma}=\left.\mu^{-1} \mathbf{n} \cdot \nabla p\right|_{\Gamma}$, since $\left.\mathbf{v}\right|_{\Gamma}=0$.

It is clear that in the case $\left.\mathbf{n} \cdot \nabla p\right|_{\Gamma} \neq 0$ there exists no $\mathbf{w}$ such that $\left.\mathbf{n} \cdot \mathbf{w}\right|_{\Gamma}=0$.

b) If $p$ satisfies Neumann condition 82 , then $\nabla p=0$ and $\mathbf{w}=\mathbf{v}$.

In the case $G=B, \mathbf{v}$ is an element of the space $\mathcal{B}$, since $\operatorname{div} \mathbf{v}=0$ and $\left.\mathbf{n} \cdot \mathbf{v}\right|_{S}=0$. We represent $\mathbf{v} \in \mathcal{B}$ as the series

$$
\mathbf{v}=\sum_{n=1}^{\infty} \sum_{m=1}^{\infty} \sum_{k=-n}^{n}\left[\left(\mathbf{v}, \mathbf{q}_{n, m, k}^{+}\right) \mathbf{q}_{n, m, k}^{+}(\mathbf{x})+\left(\mathbf{v}, \mathbf{q}_{n, m, k}^{-}\right) \mathbf{q}_{n, m, k}^{-}(\mathbf{x})\right]
$$

and substitute the series into the equation. We obtain the identity

$$
\begin{gathered}
(\operatorname{rot}+\lambda \mathrm{I})(\operatorname{rot}-\lambda \mathrm{I}) \mathbf{v}= \\
=\sum_{n=1}^{\infty} \sum_{m=1}^{\infty}\left(\lambda_{n, m}^{2}-\lambda^{2}\right) \sum_{k=-n}^{n}\left[\left(\mathbf{v}, \mathbf{q}_{n, m, k}^{+}\right) \mathbf{q}_{n, m, k}^{+}(\mathbf{x})+\left(\mathbf{v}, \mathbf{q}_{n, m, k}^{-}\right) \mathbf{q}_{n, m, k}^{-}(\mathbf{x})\right]=0 .
\end{gathered}
$$

If $\lambda_{n, m}^{2}-\lambda^{2} \neq 0$ for each $n, m \in \mathcal{N}$, then $\left(\mathbf{v}, \mathbf{q}_{n, m, k}^{ \pm}\right)=0$ for each $n, m \in \mathcal{N}, k \in[-n, n]$ due to the orthogonality of the basis vectors $\mathbf{q}_{n, m, k}^{ \pm}$. The completeness of the system $\left\{\mathbf{q}_{n, m, k}^{ \pm}\right\}$in $\mathcal{B}$ yields $\mathbf{v}(\mathbf{x})=0$. But it is impossible by the assumption. Hence, there exists a pair $n^{\prime}, m^{\prime} \in \mathcal{N}$ such that $\lambda^{2}=\lambda_{n^{\prime}, m^{\prime}}^{2}$. Letting

$$
\mathbf{u}^{ \pm}(\mathbf{x})=\sum_{k=-n^{\prime}}^{n^{\prime}}\left(\mathbf{v}, \mathbf{q}_{n^{\prime}, m^{\prime}, k}^{ \pm}\right) \mathbf{q}_{n^{\prime}, m^{\prime}, k}^{ \pm}(\mathbf{x})
$$

we obtain expansion 87 . The proof is complete.

Thus, the solution of Problem 5 is reduced to finding the solutions $\left(\lambda, \mathbf{u}^{+}\right)$and $\left(-\lambda, \mathbf{u}^{-}\right)$to Problem 1 as $\lambda \neq 0$ satisfying the condition $\left.\left(\mathbf{u}^{+}+\mathbf{u}^{-}\right)\right|_{S}=0$.

6.2. Formulae for eigenfunctions of Stokes operator in ball. We let $c_{\kappa}^{ \pm}=c_{\kappa} \Phi_{n}\left(\lambda_{n, m}^{\mp} R\right)$ in formulae (37) to obtain

$$
\begin{aligned}
& \left(u_{\varphi}+i u_{\theta}\right)_{\kappa}^{+}=c_{\kappa} \Phi_{n}\left(\lambda_{n, m}^{-} R\right)\left(\lambda_{n, m}^{+} r\right)^{-1} \Phi_{n}\left(\lambda_{n, m}^{+} r\right) \mathrm{H} Y_{n}^{k}(\theta, \varphi), \\
& \left(u_{\varphi}+i u_{\theta}\right)_{\kappa}^{-}=c_{\kappa} \Phi_{n}\left(\lambda_{n, m}^{+} R\right)\left(\lambda_{n, m}^{-} r\right)^{-1} \Phi_{n}\left(\lambda_{n, m}^{-} r\right) \mathrm{H} Y_{n}^{k}(\theta, \varphi) .
\end{aligned}
$$

It follows that as $r=R$, the sum $w_{\kappa}^{+}+w_{\kappa}^{-}$vanishes for all angles $\theta$ and $\varphi$ and each complex constant $c_{\kappa}$.

The functions $\psi_{n}\left(\lambda_{n, m}^{ \pm} r\right), Y_{n}^{k}(\theta, \varphi)$ and the number $\lambda_{n, m}^{ \pm}= \pm \rho_{n, m} / R$ are real. According to (14), $\psi_{n}\left(\lambda_{n, m}^{-} r\right)=(-1)^{n} \psi_{n}\left(\lambda_{n, m} r\right)$. Hence, $\Phi_{n}\left(\lambda_{n, m}^{-} r\right)=(-1)^{n} \overline{\Phi_{n}\left(\lambda_{n, m} r\right)}$ (see Subsection 3.3, where it was proven that the number $\Phi_{n}\left(\rho_{n, m}\right)$ is real).

Hence, the radial component of the vector $\mathbf{v}_{\kappa}=\mathbf{u}_{\kappa}^{+}+\mathbf{u}_{\kappa}^{-}$disappears,

$$
\begin{aligned}
& c_{\kappa}\left(\lambda_{n, m} r\right)^{-1}\left[\Phi_{n}\left(\lambda_{n, m}^{-} R\right) \psi_{n}\left(\lambda_{n, m}^{+} r\right)-\Phi_{n}\left(\lambda_{n, m}^{+} R\right) \psi_{n}\left(\lambda_{n, m}^{-} r\right)\right] Y_{n}^{k}(\theta, \varphi) \mathbf{i}_{r}= \\
& =c_{\kappa}(-1)^{n}\left(\lambda_{n, m} r\right)^{-1}\left[\Phi_{n}\left(\rho_{n, m}\right)-\Phi_{n}\left(\rho_{n, m}\right)\right] \psi_{n}\left(\lambda_{n, m} r\right) Y_{n}^{k}(\theta, \varphi) \mathbf{i}_{r}=0,
\end{aligned}
$$


and its tangential projection is

$$
\begin{aligned}
& \operatorname{Re}\left\{c_{\kappa}(-1)^{n}\left(\lambda_{n, m} r\right)^{-1} \Phi_{n}\left(\rho_{n, m}\right)\left[\Phi_{n}\left(\lambda_{n, m} r\right)-\bar{\Phi}_{n}\left(\lambda_{n, m} r\right)\right] H Y_{n}^{k}(\theta, \varphi) \mathbf{i}_{\varphi}\right\}+ \\
& \quad+\operatorname{Im}\left\{c_{\kappa}(-1)^{n}\left(\lambda_{n, m} r\right)^{-1} \Phi_{n}\left(\rho_{n, m}\right)\left[\Phi_{n}\left(\lambda_{n, m} r\right)-\bar{\Phi}_{n}\left(\lambda_{n, m}\right] H Y_{n}^{k}(\theta, \varphi) \mathbf{i}_{\theta}\right\} .\right.
\end{aligned}
$$

The expression in square brackets is a pure imaginary quantity. Taking the constant $c_{\kappa}=i b_{\kappa}$ pure imaginary as well, $b_{\kappa} \in \mathcal{R}$, we obtain the vector function $\mathbf{v}_{\kappa}=\mathbf{u}_{\kappa}^{+}+\mathbf{u}_{\kappa}^{-}$which is represented as the sum of two orthogonal vectors

$$
\begin{gathered}
\mathbf{v}_{\kappa}=b_{\kappa} \Phi_{n}\left(\rho_{n, m}\right)\left(\lambda_{n, m} r\right)^{-1} \operatorname{Im}\left[\Phi_{n}\left(\lambda_{n, m} r\right)\right] \\
\left(\operatorname{Re} H Y_{n}^{k}(\theta, \varphi) \mathbf{i}_{\varphi}+\operatorname{Im} H Y_{n}^{k}(\theta, \varphi) \mathbf{i}_{\theta}\right) .
\end{gathered}
$$

Thus, $\mathbf{v}_{\kappa}=\mathbf{u}_{\kappa}^{+}+\mathbf{u}_{\kappa}^{-}$is a real vector eigenfunction for the Stokes operator associated with the eigenvalue $\nu \lambda_{n, m}^{2}$. Normalizing vector functions $\mathbf{u}_{\kappa}^{ \pm}$in $\mathbf{L}_{2}(B)$, we obtain the vector eigenfunctions for the Stokes operator in the form $\mathbf{v}_{\kappa}=\mathbf{q}_{\kappa}^{+}+\mathbf{q}_{\kappa}^{-}$. Hence, we have proven

Theorem 6. The eigenvalues $\mu_{n, m}$ of Problem 5 in a ball $B$ are equal to $\nu \lambda_{n, m}^{2}$, where $\lambda_{n, m}=\rho_{n, m} R^{-1}, R$ is the radius of the ball, and the numbers $\rho_{n, m}$ are the zeroes of the functions $\psi_{n}(z), m, n \in \mathbb{N}$.

At that, $p_{\kappa}=$ const, and the associated vector eigenfunctions $\mathbf{v}_{\kappa}$ of the Stokes operator are the sums $\mathbf{q}_{\kappa}^{+}+\mathbf{q}_{\kappa}^{-}$of the vector eigenfunctions for the curl operator.

In the spherical coordinates they are represented as sum (93) of two orthogonal vectors.

The vector functions $\mathbf{v}_{\kappa}=\mathbf{q}_{\kappa}^{+}+\mathbf{q}_{\kappa}^{-}$belong to the space $\mathbf{J}^{0}(B)$ that is the closure of compactly supported infinitely differentiable solenoidal vector functions $\dot{J}(B)$ in $\mathbf{L}_{2}(B)$ 2]. These vector functions form an orthogonal system in $\mathbf{L}_{2}(B)$ since the systems $\left\{\mathbf{q}_{\kappa}^{+}\right\},\left\{\mathbf{q}_{\kappa}^{-}\right\}$are orthonormalized.

The system $\left\{\mathbf{v}_{\kappa}\right\}$ is complete in $\mathbf{J}^{0}(B) \subset \mathcal{B}$ and the expansion of a vector function $\mathbf{g}(\mathbf{x}) \in$ $\mathbf{J}^{0}(B)$ is as follows,

$$
\mathbf{g}=1 / 2 \sum_{n=1}^{\infty} \sum_{m=1}^{\infty} \sum_{k=-n}^{n}\left(\mathbf{g}, \mathbf{v}_{n, m, k}\right) \mathbf{v}_{n, m, k}(\mathbf{x}),
$$

where the summation of series (94) is w.r.t. $n, m$ obeying $0<\rho_{n, m}<N$ and $N \rightarrow \infty$.

\section{Solving of boundary value problem (2), (3)}

By the Fourier method, one can easily solve the following boundary value problem.

Problem 6. Given a vector function $\mathbf{f}(\mathbf{x}) \in \mathcal{M}_{\mathcal{R}}$, in $\mathbf{H}^{1}(B)$, find a vector function $\mathbf{u}(\mathbf{x})$ such that

$$
\begin{gathered}
\operatorname{rot} \mathbf{u}+\lambda \mathbf{u}=\mathbf{f} \quad \text { in } B, \\
\left.\mathbf{n} \cdot \mathbf{u}\right|_{S}=0,
\end{gathered}
$$

where $\mathbf{n} \cdot \mathbf{u}$ is the projection of the vector $\mathbf{u}$ on the outward normal $\mathbf{n}$.

By $\mathbf{E}^{s}(B)$ or by $\mathbf{H}_{\text {div }}^{s}(B)$ one denotes [8] the following subspaces in $\mathbf{L}_{2}(B)$,

$$
\mathbf{E}^{s}(B)=\left\{\mathbf{v} \in \mathbf{H}^{s}(B): \operatorname{div} \mathbf{v} \in H^{s}(B), \quad\|\mathbf{v}\|_{\mathbf{E}^{s}}=\left(\|\mathbf{v}\|_{\mathbf{H}^{s}}^{2}+\|\operatorname{div} \mathbf{v}\|_{H^{s}}^{2}\right)^{1 / 2}\right\},
$$

where $s \geq 0$ are integer numbers. They are complete Hilbert spaces and

$$
\mathcal{D}(\bar{B}) \subset \mathbf{E}^{s}(B), \quad \mathbf{H}^{s+1}(B) \subset \mathbf{E}^{s}(B) \subset \mathbf{H}^{s}(B) .
$$

The quantity $\left.\mathbf{n} \cdot \mathbf{v}\right|_{S}$ is well-defined for a vector function $\mathbf{v}(\mathbf{x}) \in \mathbf{E}^{0}(B)$.

Let us give the solution to the problem in two cases. 
7.1. Solving of boundary value problem $(95),(96)$ as $\lambda \neq S p(r o t)$.

Theorem 7. If $\lambda \neq 0, \pm \lambda_{n, m}, n, m \in \mathbf{N}, \mathbf{f} \in \mathbf{E}^{0}(B)$, and $\left.\mathbf{n} \cdot \mathbf{f}\right|_{S}=0$, then the unique solution $\mathbf{u}$ to Problem 6 is given by the sum of the series $\mathbf{u}_{1}+\mathbf{u}_{2}$, where

$$
\begin{gathered}
\mathbf{u}_{1}=\lambda^{-1} \sum_{n=0}^{\infty} \sum_{m=1}^{\infty} \sum_{k=-n}^{n}\left(\mathbf{f}, \mathbf{q}_{n, m, k}\right) \mathbf{q}_{n, m, k}(\mathbf{x}), \\
\mathbf{u}_{2}=\sum_{n=1}^{\infty} \sum_{m=1}^{\infty} \sum_{k=-n}^{n}\left[\left(\lambda+\lambda_{n, m}\right)^{-1}\left(\mathbf{f}, \mathbf{q}_{n, m, k}^{+}\right) \mathbf{q}_{n, m, k}^{+}(\mathbf{x})+\left(\lambda-\lambda_{n, m}\right)^{-1}\left(\mathbf{f}, \mathbf{q}_{n, m, k}^{-}\right) \mathbf{q}_{n, m, k}^{-}(\mathbf{x})\right] .
\end{gathered}
$$

The solution belongs to the Sobolev space $\mathbf{H}^{1}(B)$.

If $\mathbf{f} \in \mathcal{A}=\left\{\nabla h: h \in H^{1}(B)\right\}$, the operator $\mathbf{u}=\lambda^{-1} \mathbf{f}$ maps $\mathcal{A}$ onto $\mathcal{A}$.

If $\mathbf{f} \in \mathcal{B} \perp \mathcal{A}$ in $\mathbf{L}_{2}(B)$, then $\mathbf{u}=\mathbf{u}_{2}$ maps $\mathcal{B}$ into $\mathbf{H}^{1}(B)$.

If $\mathbf{f} \in C_{0}^{\infty}(B), \mathbf{u}$ is a classical solution to the problem belonging to $C^{\infty}(\bar{B})$.

Proof. Formulae (99) can be obtained in various ways. For instance, assuming that $\mathbf{u}$ and $\mathbf{f}$ in equation (95) belong to the space $\mathcal{D}(B)$, we multiply both sides by $\mathbf{q}_{n, m, k}(\mathbf{x})$ (respectively, by $\left.\mathbf{q}_{n, m, k}^{ \pm}(\mathbf{x})\right)$ and integrate by parts. The uniqueness of the solution follows from the completeness of the eigenfunctions of the curl operator in $\mathbf{L}_{2}(B)$.

If $\mathbf{f} \in \mathcal{D}(B)$, in accordance with Subsection 5.3, series $(99), 100$ converge in each of the spaces $\mathbf{H}^{s}(B), s=1,2, \ldots$ and give the classical solution to the problem.

If $\mathbf{f} \in \mathcal{A} \subset \mathbf{L}_{2}(B)$, in accordance with Subsection 5.3, we have $\mathbf{b}=0$ and thus, $\mathbf{u}_{2}=0$ and $\mathbf{u}_{1}=\lambda^{-1} \mathbf{f}$. In this case, solving of the problem is reduced to the multiplication of $\mathbf{f}$ by $\lambda^{-1}$.

If $\mathbf{f} \in \mathcal{B} \perp \mathcal{A}$ in $\mathbf{L}_{2}(B)$, in accordance with Subsection $5.3, \mathbf{a}=0, \mathbf{b}=\mathbf{f}$ and the series $\mathbf{u}_{1}$ thus disappears and $\mathbf{u}_{2}$ is determined by series $(100)$. This series converges in $\mathbf{L}_{2}(B)$ since the numbers $\left|\lambda \pm \lambda_{n, m}\right|^{-1}$ tends to zero as $\lambda_{n, m} \rightarrow \infty$. The space $\mathbf{L}_{2}(B)$ is embedded into the distributions space $\mathcal{D}^{\prime}(B)$, where series 100 can be differentiated term by term. Applying the operator rot term by term, we obtain the series

$$
\operatorname{rot} \mathbf{u}_{2}=\sum_{n=1}^{\infty} \sum_{m=1}^{\infty} \sum_{k=-n}^{n}\left[\frac{\lambda_{n, m}}{\lambda+\lambda_{n, m}}\left(\mathbf{f}, \mathbf{q}_{n, m, k}^{+}\right) \mathbf{q}_{n, m, k}^{+}(\mathbf{x})-\frac{\lambda_{n, m}}{\lambda-\lambda_{n, m}}\left(\mathbf{f}, \mathbf{q}_{n, m, k}^{-}\right) \mathbf{q}_{n, m, k}^{-}(\mathbf{x})\right]
$$

converging in $\mathbf{L}_{2}(B)$. Moreover, by the construction, the partial sums $\mathbf{S}_{j} u$ of series 100 satisfy the relations $\operatorname{div} \mathbf{S}_{j} u=0$ and $\left.\mathbf{n} \cdot \mathbf{S}_{j} u\right|_{S}=0$. Therefore, $\operatorname{div} \mathbf{u}_{2}=0$ and $\left.\mathbf{n} \cdot \mathbf{u}_{2}\right|_{S}=0$ as distributions. In accordance with Subsection 5.3, series $(100)$ converges in $\mathbf{H}^{1}(B)$.

Applying the operator rot $+\lambda \mathbf{I}$ to this series, we obtain the expansion of the vector function $\mathbf{f}(\mathbf{x}) \in \mathcal{B}$. Hence, this series is a generalized solution to Problem 6 .

In the general case, as $\mathbf{f} \in \mathbf{E}^{0}(B)$ and $\left.\mathbf{n} \cdot \mathbf{f}\right|_{S}=0$, series $(99)$ also belongs to $\mathbf{H}^{1}(B)$. Since $\operatorname{div} \mathbf{q}_{n, m, k}=\Delta g_{n, m, k}=-\left(\alpha_{n, m} / R\right)^{2} g_{n, m, k}$ and $\left\|\left(\alpha_{n, m} / R\right) g_{n, m, k}\right\|=1$, we have

$$
\begin{gathered}
\operatorname{div} \mathbf{u}_{1}=\lambda^{-1} \sum_{n=0}^{\infty} \sum_{m=1}^{\infty} \sum_{k=-n}^{n}\left(\mathbf{f}, \mathbf{q}_{n, m, k}\right) \triangle g_{n, m, k}(\mathbf{x})= \\
\lambda^{-1} \sum_{n=0}^{\infty} \sum_{m=1}^{\infty} \sum_{k=-n}^{n}\left(\operatorname{div} \mathbf{f}, g_{n, m, k}\right)\left(\alpha_{n, m} / R\right)^{2} g_{n, m, k}(\mathbf{x})=\lambda^{-1} \operatorname{div} \mathbf{f}
\end{gathered}
$$

Therefore, the sum of series $(99)$ and $(100)$ is the solution to Problem 6. The proof is complete. 


\subsection{Solving of Problem 6 as $\lambda=0$.}

Theorem 8. If $\lambda=0, \quad \mathbf{f} \in \mathbf{E}^{0}(B)$ and $\left.\mathbf{n} \cdot \mathbf{f}\right|_{S}=0$, then Problem 6 is solvable in $\mathbf{L}_{2}(B)$ if and only if $\operatorname{div} \mathbf{f}=0$. The homogeneous problem has infinitely many linearly independent solutions,

$$
\mathbf{u}_{0}=\sum_{n=0}^{\infty} \sum_{m=1}^{\infty} \sum_{k=-n}^{n} \xi_{n, m, k} \mathbf{q}_{n, m, k}(\mathbf{x}),
$$

where $\xi_{n, m, k}$ are arbitrary constants such that $\mathbf{u}_{0} \in \mathbf{L}_{2}(B)$.

The general solution to the inhomogeneous problem reads as $\mathbf{u}_{0}+G_{0}^{+} \mathbf{f}+G_{0}^{-} \mathbf{f}$, where

$$
G_{0}^{ \pm} \mathbf{f} \equiv \pm \sum_{n=1}^{\infty} \sum_{m=1}^{\infty} \sum_{k=-n}^{n} \lambda_{n, m}^{-1}\left(\mathbf{f}, \mathbf{q}_{n, m, k}^{ \pm}\right) \mathbf{q}_{n, m, k}^{ \pm}(\mathbf{x}), \quad G_{0}^{ \pm} \mathbf{f} \in \mathbf{H}^{1}(B) .
$$

If $\xi_{n, m, k}$ are such that $\mathbf{u}_{0} \in \mathbf{H}^{1}(B)$, the solution of the problem belongs to $\mathbf{H}^{1}(B)$.

Proof. The necessity of the condition $\operatorname{div} \mathbf{f}=0$ is obvious, while the sufficiency follows from the identity $\operatorname{div} \mathbf{u}_{1}=\lambda^{-1} \operatorname{div} \mathbf{f}$. The relations $G_{0}^{ \pm} \mathbf{f} \in \mathbf{H}^{1}(B)$ were proven in Subsection 7.1. Next, $\operatorname{rot} \mathbf{u}_{0}=0$, if $\mathbf{u}_{0} \in \mathbf{H}^{1}(B)$, and $\operatorname{rot}\left(G_{0}^{+} \mathbf{f}+G_{0}^{-} \mathbf{f}\right)=\mathbf{f}$. The proof is complete.

\section{BIBLIOGRAPHY}

1. O.A. Ladyzhenskaya. Construction of bases in spaces of solenoidal vector fields // Zapiski nauchnych seminarov POMI. 2003. V. 306. P. 71-85. [J. Math. Sci. 2005. V. 130, No. 4. P. 4827-4835.]

2. O.A. Ladyzhenskaya. Mathematical problems of dynamics of viscous incompressible liquids. Nauka, Moscow. 1970.

3. V.S. Vladimirov. Equations of mathematical physics. Nauka, Moscow. 1988. [Marcel Dekker, Inc., New York. 1971.]

4. V.V. Kozlov. Dynamical Systems X: General Theory of Vortices. "Udmurtskii universitet", Izhevsk. 1998. [Encyclopaedia of Mathematical Sciences, V. 67. Springer, New York. 2003.]

5. V.P. Mikhailov. Partial differential equations. Nauka, Moscow. 1975. (in Russian.)

6. B.R. Vaŭnberg, V.V. Grušin. Uniformly nonelliptic problems. I. // Matem. sbornik. 1967. V. 72(114), No. 4. P. 602-636. [Sb. Math. 1967. V. 1, No. 4. P. 543-568.]

7. V.A. Solonnikov. Overdetermined elliptic boundary value problems // Zapiski nauchnych seminarov LOMI. 1971. V. 21, No. 5. P. 112-158. (in Russian).

8. R. Temam. Navier-Stokes equations. Theory and numerical analysis. North-Holland Publishing Company, New York. 1977.

9. V.V. Pukhnachov. Symmetries in NavierStokes equations // Uspekhi mekhaniki. 2006. No. 6. P. 3-76. (in Russian.)

10. H. Weil. The method of orthogonal projection in potetial theory // Duke Math. J. 1940. V. 7, No. 1. P. 411-444.

11. S. Chandrasekhar. On force-free magnetic fields // Proc. Nat. Ac. Sci. 1956. V. 42. No. 1. P. 1-5.

12. J.B. Taylor. Relaxation of toroidal plasma and generation of reverse magnetic fields // Phys. Rev. Letters. 1974. V. 33, No. 19. P. 1139-1141.

13. V.I. Arnold. Sur la topologie des écoulements stationnaires des fluides parfaits // P. R. Acad. Sci. Paris. 1965. V. 261, No. 1. P. 17-20.

14. M. Henon. Sur la topologie des lignes de courant dans un case particulier // C. R. Acad. Sci. Paris. 1966. V. 262, No. 1. P. 312-314.

15. R.S. Saks. Spectral problems for the curl and Stokes operators // Doklady AN. 2007. V. 416, No. 4, P. 446-450. [Dokl. Math. 2007. V. 76, No. 2. P. 724-728.]

16. R.S. Saks. Solution of the spectral problem for the curl and Stokes operators with periodic boundary conditions // Zapiski nauchnych seminarov POMI. 2004. V. 318. P. 246-276. [J. Math. Sci. 2006. V. 136, No. 2. P. 3794-3811.]

17. R.S. Saks. On the boundary value problems for the system rot $u+\lambda u=h / /$ Dokl. Akad. Nauk SSSR. 1971. V. 199, No. 5. P. 1022-1025. [Sov. Math. Dokl. 1971. V. 12. P. 1240-1244.] 
18. R.S. Saks. Boundary-value problems for the system rot $u+\lambda u=h / /$ Differ. Uravn. 1972. V. 8. No. 1. P. 126-140. [Differ. Equat. 1972. V. 8. P. 97-102.]

19. A.A. Fursenko. Boundary value for one unifomly non-elliptic system. Master's diploma, NSU, Novosibirsk. 1971. (in Russian.)

20. R.S. Saks. Boundary value problems for elliptic systems of differential equaions. NSU, Novosibirsk. 1975. (in Russian.)

21. R.S. Saks. Spectrum of the curl operator in ball subject to non-penetration condition and eigenvalues of oscillations of elastic ball clamped on boundary // Proceedings of international conference "Complex analysis, differential equations and related questions". IV. Applied mathematics. Ufa, IM CS USC RAS. 2000. P. 61-68. (in Russian.)

22. S. Chandrasekhar, P.S. Kendall On force-free magnetic fields // Astrophys. J. 1957. V. 126, No. 2. P. 457-460.

23. D. Montgomery, L. Turner, G. Vahala Three-dimentional magnetohydrodyamic turbulence in cylindrical geometry // Phys. Fluids. 1978. V. 21. No. 5. P. 757-764.

24. P.E. Berhin. A selfadjoint boundary-value problem for the system $* d u+\lambda u=f / /$ Dokl. Akad. Nauk SSSR. 1975. V. 222, No. 1. P. 15-17. [Sov. Math. Dokl. 1975. V. 16. P. 557-559.]

25. Y. Giga, Z. Yoshida. Remark on spectra of operator rot // Math. Z. 1990. V. 204, No. 1. P. 235-245.

26. R. Picard. On selfadjoint realization of curl and some its applications // Preprint: Technische Universität Dresden: MATH-AN-02-96. Dresden, Marz. 1996.

27. R.S. Saks. On properties of the generalized elliptic pseudo-differential operators on closed manifolds // Zapiski nauchnych seminarov POMI. 1997. V. 243. P. 215-269. [J. Math. Sci. 2000. V. 99, No. 1. P. 936-968.]

28. A.S. Makhalov, V.P. Nikolaenko. Global solubility of the three-dimensional Navier-Stokes equations with uniformly large initial vorticity // Uspekhi matem. nauk. 2003. 2003. V. 58. No. 2. P. 79-93. [Russ. Math. Surv. 2003. V. 58, No. 2. P. 287-318.]

29. R.S. Saks. Global solutions of the Navier-Stokes equations in a uniformly rotating space // Teor. Matem. Fiz. 2010. V. 162, No. 2. P. 196-215. [Theor. Math. Phys. 2010. V. 162, No. 2. P. 163-178.]

30. R.S. Saks. Cauchy problem for navier-stokes equations, Fourier method // Ufimskij matem. zhur. 2011. V. 3. No. 1. P. 53-79. [Ufa Math. J. 2011. V. 3. No. 1. P. 51-77.]

Romen Semenovich Saks

Institute of Mathematics CS USC RAS,

Chernyshevskii str., 112,

450077, Ufa, Russia

E-mail: romen-saks@yandex.ru 